\title{
Transglutaminase mediated asprosin oligomerization allows its tissue storage as fibers
}

Yousef A.T. Morcos ${ }^{1,2}$, Galyna Pryymachuk ${ }^{3}$, Thorben Hoffmann ${ }^{2}$, Steffen Lütke ${ }^{1,2}$, Antje Gerken ${ }^{1,2}$, Nadin Piekarek ${ }^{3}$, Margarete Odenthal ${ }^{4,5}$, Uta Drebber ${ }^{4}$, Wilhelm Bloch ${ }^{6}$, Bert Callewaert ${ }^{7,8}$, Mats Paulsson ${ }^{1,5,9}$, Eva Hucklenbruch-Rother ${ }^{2}$, Gerhard Sengle ${ }^{1,2,5,9^{*}}$

${ }^{1}$ Center for Biochemistry, Faculty of Medicine and University Hospital of Cologne, University of Cologne, Joseph-Stelzmann-Street 52, 50931 Cologne, Germany

${ }^{2}$ Department of Pediatrics and Adolescent Medicine, Faculty of Medicine and University Hospital Cologne, University of Cologne, Cologne, Germany

${ }^{3}$ Department of Anatomy I, Faculty of Medicine and University Hospital Cologne, University of Cologne, Cologne, Germany

${ }^{4}$ Institute of Pathology, Faculty of Medicine and University Hospital Cologne, University of Cologne, Cologne, Germany

${ }^{5}$ Center for Molecular Medicine Cologne (CMMC), University of Cologne, Cologne, Germany ${ }^{6}$ Institute of Cardiology and Sports Medicine, German Sport University Cologne, Cologne, Germany ${ }^{7}$ Center for Medical Genetics Ghent, Ghent University Hospital, Ghent 9000, Belgium

${ }^{8}$ Department of Biomolecular Medicine, Ghent University, Ghent 9000, Belgium

${ }^{9}$ Cologne Center for Musculoskeletal Biomechanics (CCMB), Faculty of Medicine and University Hospital of Cologne, University of Cologne, Cologne, Germany

*corresponding author: Gerhard Sengle

Email: gsengle@uni-koeln.de

Running title: Asprosin oligomerization by transglutaminase activity

Keywords: Asprosin, transglutaminase 2, fibrillin, extracellular matrix, protein oligomerization, connective tissues 


\begin{abstract}
Asprosin, the C-terminal furin cleavage product of profibrillin-1, was reported to act as a hormone that circulates at nanomolar levels and is recruited to the liver where it induces G protein-coupled activation of the cAMP-PKA pathway and stimulates rapid glucose release into the circulation. Although derived from profibrillin-1, a multidomain extracellular matrix glycoprotein with a ubiquitous distribution in connective tissues, little is known about the tissue distribution of asprosin. In the current view, asprosin is mainly produced by white adipose tissue from where it is released into the blood in monomeric form. Here, by employing newly generated specific asprosin antibodies we monitored the distribution pattern of asprosin in human and murine connective tissues such as placenta, and muscle. Thereby we detected the presence of asprosin positive extracellular fibers. Further, by screening established cell lines for asprosin synthesis we found that most cells derived from musculoskeletal tissues render asprosin into an oligomerized form. This oligomerization is facilitated by transglutaminase activity and requires an intact fibrillin fiber network for proper linear deposition. Our data suggest a new extracellular storage mechanism of asprosin in oligomerized form which may regulate its cellular bioavailability in tissues.
\end{abstract}




\section{Introduction}

Fibrillin-1 is a large, $350 \mathrm{kDa}$ extracellular matrix (ECM) glycoprotein that when mutated leads to the multi-system disorder Marfan syndrome (MFS; OMIM\#154700) affecting mostly the musculoskeletal (e.g. long bone overgrowth, muscle wasting, hyperflexible joints) and cardiovascular system (e.g. aortic root aneurysm formation). The multiple clinical features of MFS reflect the ubiquitous tissue distribution pattern of fibrillin-1 (1). Since most mutations in FBN1 result in a slender habitus with little subcutaneous fat (2), it is intuitive to speculate that fibrillin-1 plays a role in metabolism and body fat formation. In addition, specific mutations within exon 64 at the 3 ' end of FBN1 were described to lead to marfanoid-progeroid-lipodystrophy syndrome (MFLS; OMIM\#616914) (3), a fibrillinopathy characterized by progeroid facial features and severe lipodystrophy (3-6). A mechanistic explanation for these striking clinical features was provided by the discovery that the Cterminal cleavage product of profibrillin-1 serves as a fasting-induced glucogenic protein hormone that modulates hepatic glucose release (7). Profibrillin is translated as a 2,871-amino-acid long proprotein, which is cleaved at the C-terminus by the protease furin (8). In addition to mature fibrillin-1, this cleavage generates a 140-amino-acid long C-terminal cleavage product. MFLS mutations were found to be clustered around the furin cleavage site, thereby causing heterozygous ablation of the C-terminal cleavage product which was only detectable at strongly reduced levels in plasma of patients (7). This C-terminal furin cleavage product of fibrillin-1 was termed "asprosin" after the Greek word for white because of its strong impact on white fat tissue (WAT) (7).

Asprosin was reported to circulate at nanomolar levels and is recruited to the liver where it induces $\mathrm{G}$ protein-coupled activation of the cAMP-PKA pathway and stimulates rapid glucose release into the circulation (7). Following a circadian rhythm and triggered by fasting, asprosin induces hepatic glucose release via the G-protein-coupled OLFR734 receptor (7,9) and reduces insulin secretion from pancreatic $\beta$-cells (10). Asprosin has also been shown to cross the blood brain barrier and activate hunger-stimulating AgRP (Agouti-related peptide) neurons in the hypothalamus which induces appetite in mice (11). In addition, asprosin was shown to impair insulin sensitivity in skeletal muscle cells in vitro (12). There are also several reports of increased serum asprosin levels in obese patients and patients with type 2 diabetes mellitus (T2DM) and that asprosin serum concentrations are 
positively correlated with insulin resistance (13). In mice, antibody-mediated neutralization of asprosin leads to reduced food intake and body weight as well as improved insulin sensitivity (11). Therefore, asprosin currently ranges among the most promising candidates for the pharmacological therapy of obesity, T2DM and other metabolic disorders (13).

Although derived from ubiquitously expressed profibrillin-1, little is known about the tissue distribution of asprosin. In the current view, asprosin is mainly produced by WAT from where it is released into the blood in monomeric form. However, our previous findings showed that asprosin is synthesized and secreted by connective tissue resident cells such as fibroblasts and chondrocytes (14) - a finding which supports the notion of local asprosin storage within tissue-specific microenvironments. Here, by employing newly generated specific asprosin antibodies we monitored the distribution pattern of asprosin in various human and murine connective tissues, including placenta, and muscle. In addition, we monitored the synthesis of asprosin in various established cell lines. Our data reveal new insight about how asprosin may be stored within the extracellular microenvironment of connective tissues and how this affects its cellular bioavailability.

\section{Results}

\section{Extracellular storage of asprosin as fibers in tissues}

Since fibrillin-1 shows a broad tissue distribution, we wanted to monitor asprosin synthesis and potential extracellular storage by tissues. Recently, we were able to generate a highly specific antiasprosin antibody that allowed the sensitive detection of asprosin in clinical samples (14). Our new asprosin antibody does not show any cross-reactivity to fibrillin-1 (14) and can therefore be used as reliable tool to monitor the tissue distribution of asprosin. Immunofluorescence analysis showed that asprosin is present in extra- and intracellular pools in different tissues (Fig. 1). For instance in liver, predominantly intracellular asprosin storage in vesicular structures could be observed (Fig. 1). However, in placenta and cardiac muscle, the presence of asprosin positive fibers was detected (Fig. $1)$. 


\section{Asprosin is detected as oligomers in cultures of cell lines}

To investigate asprosin synthesis and secretion by cells derived from connective tissues, we screened different cell lines for intra- and extracellular presence of asprosin by immunoblot analysis. We detected asprosin positive bands of higher molecular weight suggesting the presence of asprosin oligomers (Fig. 2A). Thereby we observed a higher abundance of asprosin oligomers in cell culture supernatants of cell lines derived from musculoskeletal tissues such as U2OS (bone osteosarcoma epithelial cells) or $\mathrm{C} 2 \mathrm{C} 12$ (mouse myoblasts) when compared to Wi26 embryonic lung fibroblasts or retinal pigment epithelial (RPE) cells (Fig. 2A). To investigate the extracellular deposition of asprosin in cell culture, we employed immunofluorescence analysis after 7-9 days of culture time. Interestingly, similar to our findings in tissues we also detected asprosin positive fibers in the ECM of certain cell lines such as 3T3-L1 fibroblasts (model for fibro/adipogenic progenitors present in skeletal muscle) as well as primary human skin fibroblasts and human chondrocytes (Fig. 2B).

\section{Overexpression of monomeric asprosin in musculoskeletal cells results in oligomerization}

To further investigate the formation of asprosin oligomerization in musculoskeletal cells, we performed transfection of 3T3-L1 cells with an overexpression construct encoding for monomeric asprosin. Western blot analysis of the collected conditioned media after SDS-PAGE analysis showed that most asprosin was detected in bands representing higher molecular weight oligomers that persisted even under reducing conditions (Fig. 3A). However, when HEK-293 cells were transfected with the same construct, only asprosin monomers were detected in the cell culture supernatant (Fig. 3A). Interestingly, signals representing oligomerized forms of asprosin were also detected in cell lysates and were resistent in the presence of reducing agents. Immunofluorescence analysis of cultured 3T3-L1 cells showed the presence of fibers consisting of endogenously expressed asprosin as well as overexpressed monomeric asprosin after transfection (Fig. 3B). 


\section{Asprosin is a substrate of tissue transglutaminase}

Resistance of asprosin oligomer bands to reducing agents indicated stable covalent linkage between monomers. Fibrillin monomers are known to be crosslinked by transglutaminase 2 (TG2) which facilitates the assembly of stable fibrillin monomers (15). In general, transglutaminases primarily catalyse the formation of an isopeptide bond between $\gamma$-carboxamide groups of glutamine residue side chains and the $\varepsilon$-amino groups of lysine residue side chains with subsequent release of ammonia. Thereby, transglutaminases are very specific for the recognition of the glutamine residue and also depend on the charge of the flanking amino acid sequence (16). Since musculoskeletal cells are known to express transglutaminases (17), we tested whether asprosin is a substrate of TG2. Addition of TG2 to monomeric asprosin resulted in significant shift of asprosin positive immunoblot signals towards higher molecular weight positions (Fig. 4A). A similar pattern of bands representing asprosin oligomers was obtained when monomeric asprosin was incubated in the presence of the chemical crosslinker DSS (Fig. 4B). To confirm that asprosin is a substrate of TG2, recombinant asprosin and the co-substrate monodansylcadaverine (MDC) were incubated with TG2. Thereby, any TG2 substrate would become covalently tethered to MDC which allows sensitive detection by UV light (Fig. 4C). Subsequent to asprosin incubation with MDC and TG2, we were able to visualize monomeric and dimeric asprosin by UV light, thereby demonstrating that asprosin is a substrate of TG2 (Fig. 4D). Since covalent crosslinking of MDC adds 335.5 Da to the molecular weight of asprosin, we wanted to identify via which glutamine residues asprosin crosslinking is mediated. Therefore, we subjected MDC tethered monomeric asprosin to a proteolytic digestion by trypsin and Lys-C and analyzed the resulting fragments by mass spectrometry analysis. Our analysis identified the glutamine residues Q99 and Q113 to be critical for TG2 mediated asprosin oligomerization (Fig. 4E).

\section{Asprosin fiber formation requires the presence of an intact fibrillin fiber network}

To understand whether asprosin fiber formation requires the presence of extracellular scaffolds, we administered recombinant asprosin to cell cultures of several cell lines and analyzed potential fiber formation by immunofluorescence analysis. Interestingly, while asprosin appeared to form aggregates in cultures of U2OS cells addition to RPE cells or primary skin fibroblast cultures resulted in robust 
formation of asprosin positive fibers that showed an overlaping distrubution to the fibronectin network

(Fig. 5). Asprosin fiber formation was also observed when we administered asprosin that was tethered to a fluorescent dye (atto-550) (supplementary Fig. S1). However, we did not observe fiber formation when we added placensin, the C-terminal propeptide of fibrilin-2 (18), to fibroblast cultures (supplementary Fig. S2C).

Since TG2 is known to establish functional crosslinks stabilizing the fibonectin and fibrillin microfibril networks $(19,20)$, we wanted to test whether TG2 may also tether asprosin to either of them. Pull down experiments revealed that asprosin interacted with the N-terminal half of fibrillin-1 and not with full length fibronectin (Fig. 6A,B). Also interaction studies employing surface plasmon resonance confirmed that asprosin interacted to the N-terminal region of fibrillin-1 and not with full length fibronectin (Fig. 6C). To test whether intact fibrillin fiber assembly is required for asprosin fiber formation we added recombinant asprosin to primary skin fibroblast cultures of MFS patients which show a compromised fibrillin fiber formation but no impact on fibronectin assembly. Asprosin fiber formation was abolished when fibrillin fiber formation was deficient (Fig. 7). Moreover, by employing newly generated asprosin antibodies specific for detection of endogenous asprosin in murine tissues (supplementary Fig. S3) we could show that asprosin co-localizes with fibrillin fibers in connective tissues such as skeletal muscle, heart, perichondrium, and WAT (Fig. 8).

\section{Discussion}

Currently, asprosin is mainly viewed as WAT derived hormone that is released into the blood stream to regulate glucose release in the liver $(7,13)$. However, since asprosin is the C-terminal propeptide of profibrillin we wondered whether it has a similar tissue distribution as fibrillin-1. By generating new asprosin specific antibodies that do not crossreact with full length fibrillin-1 (14) we were able to detect for the first time extracellular deposits of asprosin as fibers in tissues. Thereby the presence of fibers was more prominently observed in certain tissue microenvironments such as skeletal or cardiac muscle. However in liver, asprosin was mainly found in intracellular vesicles (Fig. 1) indicating its rapid utilization in this metabolically active tissue. 
Currently, the function of asprosin fibers is unknown. We hypothesize that the oligomerization and linear deposition of asprosin as fibers represents a local asprosin storage mechanism within tissuespecific microenvironments (Fig. 9). Asprosin stored in tissues may function as sensor for local energy demand. From there it may be released by specific, yet unknown activation reactions into the serum. Controlled asprosin release by tissues may be a so far unexplored mechanism how tissues signal energy demand to the metabolic system. A metabolic function of asprosin in regulation energy demand of tissue resident cells was already proposed in in vitro cell cultures. For instance, experiments with murine myoblasts $(\mathrm{C} 2 \mathrm{C} 12)$ showed that asprosin is not only able to interfere with muscle cell insulin sensitivity (21), but also up-regulates glucose transporter 4 (GLUT4) expression in $\mathrm{C} 2 \mathrm{C} 12$ myotubes and thereby enhance local glucose uptake by tissue muscle cells (22).

Interestingly, we found that asprosin is a substrate of TG2, a crosslinking enzyme that is known to facilitate fibronectin, fbrillin-1, and elastic fiber assembly $(15,19,23,24)$. TG2 is very selective regarding the glutamine residues it subjects to crosslinking (16). Our experiments showed that placensin, the C-terminal propeptide of fibrillin-2, does not form higher oligomers or fibers in fibroblast cultures upon addition, despite of the presence of several available glutamine residues (supplementary Fig. S1). Recently, placensin was described as a new placenta-derived glucogenic hormone that stimulates hepatic cAMP production, and protein kinase A (PKA) activity and glucose secretion. Also asprosin was shown to be expressed in human placenta and is elevated in the plasma of pregnant women complicated with gestational diabetes mellitus (GDM) and their offspring (umbilical blood) (25) after adjustment for maternal and neonatal clinical characteristics and lipid profiles. In placenta, asprosin fiber formation may have the function to fine tune the metabolic effects mediated by asprosin and placencin.

Our transfection experiments with HEK293 cells contructs encoding monomeric asprosin showed that asprosin oligomers were also detected intracellularly, whereas in the conditioned media only monomeric asprosin was detected. In contrast, in the lysates supernatant of 3T3-L1 cells asprosin oligomers were found intra- and extracellularly. Intracellular TG cross-linking is a complex theme as TGs do not pass through the conventional ER/Golgi route and require $\mathrm{Ca}^{2+}$ for activity. Nevertheless, despite low intracellular calcium levels, multiple transamidation and crosslinking substrates of 
intracellular TG2 have been identified $(17,26)$. This suggests that locally increased intracellular calcium and/or as yet uncharacterized interacting proteins may facilitate formation of active TG2. Our transfection studies with an overexpression construct coding for monomeric asprosin already indicated the formation of tetramers in cell lysates (Fig. 2B). However, in HEK293 cells oligomers may be either not secreted or extracellularly oligomerized asprosin was internalized. In future studies we will address not only how oligomerized asprosin is utilized by cells, but also by what specific release mechanisms asprosin may be liberated from its fibrous state.

Our data show that asprosin is specifically deposited on fibrillin fiber and not to fibronectin. This finding may implicate an intact fibrillin network in the proper extracellular storage of asprosin. Fibrillin is ubiquitously expressed in all tissues and is known to assemble into supramolecuar microfibrils that serves as targeting scaffolds for connective tissue derived growth factors such as TGF- $\beta$ and BMPs (27-31). By targeting and sequestration of asprosin in connective tissue microenvironments fibrillin may be involved in the regulation of spatio-temporal energy demand of tissue resident cells.

\section{Materials and method}

\section{Ethics statement}

The use of human specimen involved in this study was approved by the institutional review boards at the Medical Faculty of the University of Cologne, the German Sports University, and Ghent University. Written informed consent was obtained from patients in accordance with institutional review board policies. Written Informed consent was obtained from all participating probands and patients who agreed to have materials examined for research purposes. The study was conducted in accordance with the Declaration of Helsinki. Human liver tissue samples were collected from resected liver specimens from three patients with their informed and written consent according to the Biomasota code (13-091) at the University Hospital of Cologne. The study was approved by the ethics committee of the University Hospital of Cologne (18-052). Mice were sacrificed by cervical dislocation and immediately dissected for experiments. All animal procedures were conducted in compliance with protocols approved by the Committee on the Ethics of Animal Experiments of the 
Landesamt für Natur, Umwelt und Verbraucherschutz Nordrhein-Westfalen (84-02.04.2019.A326) and were in accordance with National Institutes of Health guidelines.

\section{Antibodies}

Pc-asp anti-asprosin, fibrillin-1 (rF90) polyclonal antibody, and fibrillin-1 rabbit monoclonal antibody (CPTC-FBN1-3) (DSHB, Iowa, USA) were previously described (14). Monoclonal anti-fibronectin antibody (\# F7387) was purchased from Merck Millipore (Massachusetts, USA). Mab anti-asprosin antibody (clone Birdy-1, AG-20B-0073) was purchased from AdipoGen Life Sciences Inc. (San Diego, USA). StrepMAB-Classic antibody (\#2-1507-001) was from IBA GmbH, Germany. The murine-asprosin antibody was generated against full-length mouse asprosin (supplementary Fig. S3). Recombinantly expressed mouse asprosin or human placensin were used to immunize rabbits for antibody production (Davids Biotechnologie GmbH, Regensburg, Germany). Recombinatly expressed human asprosin (14) was used for ployclonal antibody production in a rat (Pineda, Berlin, Germany). Preimmune serum samples $(0.5 \mathrm{ml})$ were obtained before the immunization and did not show crossreactivity to asprosin tested by ELISA. Antisera were affinity purified on CNBr-activated Sepharose 4B (Cytiva, Uppsala, Sweden) columns conjugated with the respective recombinantly expressed proteins $(300 \mu \mathrm{g})$ according to the manufacturer's instructions. Antibodies were eluted with $0.1 \mathrm{M}$ glycine $(\mathrm{pH} 2.5)$ and neutralized with $3 \mathrm{M}$ Tris/ $\mathrm{HCl}, \mathrm{pH}$. Eluted antibodies were concentrated by using Amicon Ultra Centrifugal Filters (cut-off: $10 \mathrm{kDa}$ ), (starting volume: $6 \mathrm{ml}$, end volume: $1 \mathrm{ml}$ ). The specificity of the raised antibodies was tested by diret ELISA, pull down experiments, as well as immunofluorescence of tissues and transfected cells (supplementary Figs. S2-S5).

\section{Expression and purification of recombinant proteins}

Human asprosin, human placensin, and the N-terminal half of fibrillin-1 (rF90, amino acids positions: $\left(\mathrm{M}^{1}-\mathrm{V}^{1527}\right)$ were produced and purified as previously described (14) (supplementary Fig. S2B). Cterminal half of fibrillin-1 (rF6, amino acid positions $\mathrm{V}^{1487}-\mathrm{H}^{2871}$ ) was overexpressed in HEK-293 EBNA cells. The rF6 overexpression construct (32) was a kind gift from Lynn Sakai, Oregon Health and Science University, Portland, OR, USA. Mouse asprosin cDNA was generated by GeneArt Strings 
DNA Fragments service (Thermo Fisher Scientific, Massachusetts, USA) including the restriction enzymes sequences for NheI at the 5'and XhoI at the 3' ends. Subcloning of the mouse asprosin cDNA sequence into modified pCEP-Pu vector, transfection and protein purification were as previously described (14).

\section{Preparation of fluorescently labelled asprosin (Asprosin-550)}

$1 \mathrm{mg}$ of ATTO 550 dye (\#AD 550-31, ATTO-TEC GmbH, Germany) was freshly prepared by dissolving it in $100 \mu 1$ DMSO directly before coupling to asprosin. $500 \mu \mathrm{g}$ of purified human asprosin was buffered exchanged into a coupling buffer (PBS containing $10 \mathrm{mM}$ sodium bicarbonate and 100 mM sodium hydroxide, $\mathrm{pH}$ 8.3) by Amicon Ultra Centrifugal Filters (cut-off: $3 \mathrm{kDa}$ ). The coupling reaction mixture was prepared by mixing the dissolved ATTO 550 dye and the buffer-exchanged asprosin in the coupling buffer to a final volume of $5 \mathrm{ml}$. The mixture was then incubated for $1 \mathrm{~h}$, protected from light at RT. The coupling reaction mixture was concentrated by using Amicon Ultra Centrifugal Filters (cut-off: $10 \mathrm{kDa}$ ), (starting volume: $5 \mathrm{ml}$, end volume: $0.3 \mathrm{ml}$ ). The concentration of fluorescently labelled asprosin (Asprosin-550) was measured by NanoDrop One (Thermo Fisher Scientific, Massachusetts, USA). The quantification was performed by measuring the protein absorbance at $280 \mathrm{~nm}$ and the ATTO-550 dye absorbance at $550 \mathrm{~nm}$. The integrity of the conjugated asprosin was additionally analyzed by SDS-PAGE and subjected to Coomassie Blue Staining (supplementary Fig. S1B). The fluorescence of the conjugated asprosin was visualized by ChemoStar Touch ECL \& Fluorescence Imager (Intas Science Imaging Instruments GmbH, Göttingen, Germany).

\section{Immunoblotting}

Cells were lysed in RIPA buffer (50 mM Tris $\mathrm{HCl}, 150 \mathrm{mM} \mathrm{NaCl}, 1.0 \%$ (v/v) NP-40, $0.5 \%$ (w/v) Sodium Deoxycholate, $1.0 \mathrm{mM}$ EDTA, $0.1 \%(\mathrm{w} / \mathrm{v})$ SDS and $0.01 \%(\mathrm{w} / \mathrm{v})$ at a $\mathrm{pH}$ of 7.4$)$ supplemented with cOmplete protease inhibitor cocktail (\#11697498001, Sigma-Aldrich, Darmstadt, Germany) and PhosSTOP phosphatase inhibitors (\#4906837001, Sigma-Aldrich, Darmstadt, Germany). Protein concentrations were measured using Pierce BCA Protein Assay Kit (\#23225, Thermo Fisher Scientific, Massachusetts, USA). For cell culture supernatants, when the cells 
confluency reached $90 \%$, the medium was changed with serum free medium and then collected after48 h. The collected serum free medium was filtered and concentrated with Amicon Ultra Centrifugal Filters (cut off: $3 \mathrm{kDa}$ ), (starting volume: $2 \mathrm{ml}$, end volume: $50 \mu \mathrm{l}$ ). Cell lysates and cell culture supernatants samples were resolved by $7.5 \%$ or $10 \%$ SDS-PAGE under reducing (with $\beta$ mercaptoethanol) or non-reducing conditions, then transferred to PVDF transfer membrane, $0.45 \mu \mathrm{m}$ (Thermo Fisher Scientific, Massachusetts, United States). Membranes were blocked with Pierce Protein-Free (TBS) Blocking Buffer (Thermo Fisher Scientific, Massachusetts, United States) for one hour, then incubated with the appropriate dilutions of primary antibodies overnight at $4{ }^{\circ} \mathrm{C}$ and then incubated with secondary antibody, mouse anti-rabbit IgG-HRP conjugate or goat anti-mouse IgGHRP conjugate, 1:5000 for $1 \mathrm{~h}$. All antibodies were diluted in blocking buffer. Signals were developed with SuperSignal West Pico PLUS Chemiluminescent Substrate (Thermo Fisher Scientific, Massachusetts, USA).

\section{Immunostaining}

Organs were immediately frozen in liquid nitrogen for protein isolation or coated with Tissue-Tek O.C.T. Compound (Sakura, The Netherlands) and frozen in ice-cold isopentane $\left(\sim-150^{\circ} \mathrm{C}\right)$ for cryosectioning. For immunofluorescence analysis of murine tissues, cryo-preserved tissues were cut into 5 $\mu \mathrm{m}$ sections by Leica CM1520 cryostat. After drying at RT for 30 min and fixation in 4\% PFA for 10 min, sections were rinsed in PBS and permeabilized with PBS-Tween $0.1 \%$ for $30 \mathrm{~min}$. Then samples were blocked with $10 \%$ goat serum for $2 \mathrm{~h}$. Sections were incubated at $4^{\circ} \mathrm{C}$ overnight in a humidified chamber with primary antibody against mouse-asprosin (polyclonal rabbit, dilution 1:200, lab-made), diluted in Antibody Diluent (Dako). As both antibodies are produced in rabbits, Invitrogen ${ }^{\mathrm{TM}}$ Alexa Fluor 555 Tyramide SuperBoost Kit (goat anti-rabbit $\operatorname{IgG}$ ) was used to costain asprosin and fibrillin-1. After being washed in PBS, sections were incubated with Poly-HRP-conjugated secondary antibody for $1 \mathrm{~h}$ at RT. Tyramide reaction was then induced with $1 \times$ Tyramide working solution for $7 \mathrm{~min}$. The reaction was stopped with a 1:11 diluted Reaction Stop Reagent for 1 min. After rinsing with PBS, tissue was blocked with $10 \%$ goat serum and incubated overnight at $4{ }^{\circ} \mathrm{C}$ with primary antibody against fibrillin-1 (fibrillin-1 polyclonal rabbit, dilution 1:1000, lab-made). After being washed in 
PBS, sections were incubated with the secondary antibody, Alexa Fluor 647 AffiniPure Donkey AntiRabbit IgG (H+L) (\#711-605-152, jackson immunoresearch, Pennsylvania, United States), 1:500 for 2 h. Sections were rinsed in PBS and embedded with an aqueous mounting medium containing DAPI (Sigma-Aldrich, Darmstadt, Germany). Negative control was conducted by omitting the primary antibodies. Images were taken at 40x magnification using the Olympus BX43 microscope (Olympus, Tokyo, Japan) equipped with a DP80 dual CCD camera. ImageJ software was used for image processing.

$10 \mu$ m-thick human liver cryosections were placed on the glass slides (Superfrost, Thermo Fisher Scientific Massachusetts, USA), air-dried for $60 \mathrm{~min}$ at $37^{\circ} \mathrm{C}$ and then rehydrated with phosphate-buffered saline (PBS) for 10 min. After permeabilization by $0.1 \%$ Triton X-100 plus $0.05 \%$ Tween-20 in PBS for 15 minutes sections were incubated in blocking buffer (5\% normal donkey serum (Dako) plus $0.05 \%$ Tween-20 in PBS) for $60 \mathrm{~min}$ at RT. Primary rabbit anti-asprosin antibody was then diluted in Antibody Dilution Buffer (\#AL120R100, DCS Diagnostics, Germany) buffer in 1:100 ratio and applied overnight at $4^{\circ} \mathrm{C}$. After washing 3 times for 5 min in PBS containing $0.05 \%$ Tween-20, specimens were incubated with Alexa Fluor 488-conjugated donkey anti-Rabbit $\operatorname{IgG}(\mathrm{H}+\mathrm{L})$ (\# R37118, ThermoScientific, Massachusetts, USA) diluted 1:300 in Antibody Dilution buffer containing DAPI $(1 \mu \mathrm{g} / \mathrm{mL})$ (\#62248. Thermo Fisher Scientific, Massachusetts, USA) for $1 \mathrm{~h}$ at RT. Finally, the specimens were washed once with PBS containing $0.05 \%$ Tween-20 for 5 min then twice with PBS 5 min each and mounted with ProLong Gold Antifade Mountant (\#P10144, Thermo Fisher Scientific Massachusetts, USA). Sections processed with the only secondary antibodies served as a control to exclude autofluorescence and non-specific binding. cOmplete protease inhibitor cocktail (one tablet per $50 \mathrm{ml}$ PBS, cOmplete ${ }^{\mathrm{TM}}$ ULTRA Tablets, Mini, EDTA-free, Roche) was added to all solutions and buffers used for previously described proceduers, except the permeabilization solution.

\section{Cell culture}

All cell lines used in the study were provided from American Type Culture Collection (ATCC). HDF (primary human dermal fibroblasts) from control as well as $\mathrm{HCH}$ (primary human chondrocytes) were established from biopsies. Cells were cultured in DMEM with $10 \%$ fetal calf serum (FCS) and 1\% 
penicillin/ streptomycin, they were grown in incubators maintained at $37^{\circ} \mathrm{C}$ and $5 \% \mathrm{CO}$. The cells were fed twice per week and split regularly.

\section{Surface plasmon resonance}

SPR experiments were performed as described previously $(10,13)$ using a BIAcore 2000 system (BIAcore AB, Uppsala, Sweden). Recombinant human asprosin (\#761902, Biolegend, San Diego, CA, USA) was immobilized at 1000 RUs to a CM5 sensor chip using the amine coupling kit following the manufacturer's instructions (Cytiva, Uppsala, Sweden). Interaction studies were performed by injecting 0-320 nM recombinant fibrillin-1 fragments (Start-EGF, or rF90), or fibronectin in HBS-EP buffer (0.01 M HEPES, pH 7.4, 0.15 M NaCl, 3 mM EDTA, 0.005\% (v/v) surfactant P20) (Cytiva, Uppsala, Sweden). Kinetic constants were calculated by nonlinear fitting (1:1 interaction model with mass transfer) to the association and dissociation curves according to the manufacturer's instructions (BIAevaluation version 3.0 software). Apparent equilibrium dissociation constants (KD values) were then calculated as the ratio of $\mathrm{kd} / \mathrm{ka}$.

\section{Crosslinking by transglutaminase 2 (TG2) and fluorescent-monodansylcadaverine}

$20 \mu \mathrm{g}$ of purified asprosin $(5 \mu \mathrm{g} / \mu \mathrm{l})$ was buffer exchanged by Amicon Ultra Centrifugal Filters (cut off: $3 \mathrm{kDa}$ ), with PBS containing $5 \mathrm{mM} \mathrm{CaCl} 2$ and treated with $2 \mu \mathrm{g}$ transglutaminase 2 (\#T5398, from guinea pig liver, Sigma-Aldrich, Darmstadt, Germany) (enzyme: substrate ratio, 1:10 (w/w)) in a total volume of $300 \mu 1$. The reaction mixture was incubated at $37^{\circ} \mathrm{C} .15 \mu 1$ of the reaction mixture was withdrawn after 5,10 , and $15 \mathrm{~min}$. The reaction was directly stopped by adding $5 \mu \mathrm{l}$ of Laemmli Buffer and the samples were analyzed by SDS-PAGE and western blot. For the control experiment tTG was omitted or samples were treated with $0.25 \mathrm{M}$ EDTA to sequester calcium ions and inhibit tTG activity. Fluorescent-monodansylcadaverine (MDC) (\# D4008, Aldrich, Darmstadt, Germany) was used as amine donor and acceptor sites for tissue transglutaminase enzyme activity detection as previously suggested and described (33). MDC was dissolved in DMSO, $2 \mathrm{mg}$ in $1.5 \mathrm{ml}$ DMSO to obtain a stock solution of $4 \mathrm{mM}$ MDC. $40 \mu \mathrm{g}$ of purified asprosin $(5 \mu \mathrm{g} / \mu \mathrm{l})$ was buffered exchanged with PBS containing $5 \mathrm{mM} \mathrm{CaCl} 2$ and $40 \mu \mathrm{M}$ MDC in a total volume of $120 \mu$ l. the reaction mixture was then treated with $4 \mu \mathrm{g}$ tTG $(1 \mu \mathrm{g} / \mu \mathrm{l})$ and incubated at $37^{\circ} \mathrm{C} .15 \mu \mathrm{l}$ of the reaction mixture was 
withdrawn after $5,10,15,20,30$, and $45 \mathrm{~min}$, then the reaction was directly stopped by adding $5 \mu 1$ Laemmli Buffer subjected to SDS-PAGE. The gel was then fixed 25\% isopropanol,10\% acetic acid and photographed on a $300 \mathrm{~nm}$ UV, then subjected to Coomassie Blue Staining.

\section{DSS (disuccinimidyl suberate) crosslinking}

DSS (\#21655, Thermo Fisher Scientific Massachusetts, USA) is a water-insoluble chemical crosslinker and was dissolved in dimethyl sulfoxide (DMSO), $1 \mathrm{mg}$ in 55.29 $\mu 1$ DMSO to obtain 800 $\mu$ M DSS, then serially diluted 1:2 in DMSO to obtain following DSS concentrations 400, 200, and 100 $\mu \mathrm{M}$ DSS. Cross-linking reactions were conducted in total volume $15 \mu \mathrm{l}$ containing $1 \mu \mathrm{g}$ asprosin $(0.5 \mu \mathrm{g} / \mu \mathrm{l}), 1.5 \mu \mathrm{l}$ of freshly prepared DSS to obtain final DSS concentrations $(80,40,20$, and $10 \mu \mathrm{M})$ and complete to the $15 \mu 1$ final volume with PBS. The reaction mixtures were incubated at RT for 30 min was directly stopped by adding $5 \mu 1$ of Laemmli Buffer and the samples were analyzed by SDSPAGE and western blot. For the control experiment, $1.5 \mu 1$ of DMSO was used instead of DSS.

\section{Acknowledgments}

We would like to thank the CECAD Cologne Proteomics Facility for conducting mass spectrometry analysis. Funding for this study was provided by the Deutsche Forschungsgemeinschaft (DFG, German Research Foundation) FOR2722/ B1 to M.P., and FOR2722/ M2 to E.H.R., and G.S. B.C. is a senior clinical investigator of the Research Foundation Flanders. This work was supported by a grants of the Special Research Fund of Ghent University (grant 01N04516C and BOF21/GOA/019 to B.C.). The Ghent University Hospital is a member of the European Reference Network for Skin Disorders (ERN-Skin).

\section{Author contributions}

G. Sengle designed research. Y. A. T. Morcos, G. Pryymachuk, T. Hoffmann, S. Lütke, A. Tenbieg, and N. Piekarek performed research. Y. A. T. Morcos, G. Pryymachuk, T. Hoffmann, and S. Lütke 
analyzed data. W. Bloch, M. Odenthal, U. Drebber, and B. Callewaert provided essential reagents. M.

Paulsson provided expert advise. Y. A. T. Morcos, and G. Sengle wrote the manuscript.

\section{Conflict of interest}

The authors declare no conflict of interest.

\section{References}

1. Sakai, L. Y., Keene, D. R., and Engvall, E. (1986) Fibrillin, a new 350-kD glycoprotein, is a component of extracellular microfibrils. J Cell Biol 103, 2499-2509

2. Sakai, L. Y., Keene, D. R., Renard, M., and De Backer, J. (2016) FBN1: The disease-causing gene for Marfan syndrome and other genetic disorders. Gene 591, 279-291

3. Passarge, E., Robinson, P. N., and Graul-Neumann, L. M. (2016) Marfanoid-progeroidlipodystrophy syndrome: a newly recognized fibrillinopathy. Eur J Hum Genet 24, 1244-1247

4. Graul-Neumann, L. M., Kienitz, T., Robinson, P. N., Baasanjav, S., Karow, B., GillessenKaesbach, G., Fahsold, R., Schmidt, H., Hoffmann, K., and Passarge, E. (2010) Marfan syndrome with neonatal progeroid syndrome-like lipodystrophy associated with a novel frameshift mutation at the 3 ' terminus of the FBN1-gene. Am J Med Genet A 152A, 2749-2755

5. Takenouchi, T., Hida, M., Sakamoto, Y., Torii, C., Kosaki, R., Takahashi, T., and Kosaki, K. (2013) Severe congenital lipodystrophy and a progeroid appearance: Mutation in the penultimate exon of FBN1 causing a recognizable phenotype. Am J Med Genet A 161A, 30573062

6. Jacquinet, A., Verloes, A., Callewaert, B., Coremans, C., Coucke, P., de Paepe, A., Kornak, U., Lebrun, F., Lombet, J., Pierard, G. E., Robinson, P. N., Symoens, S., Van Maldergem, L., and Debray, F. G. (2014) Neonatal progeroid variant of Marfan syndrome with congenital lipodystrophy results from mutations at the 3' end of FBN1 gene. Eur J Med Genet 57, 230 234

7. Romere, C., Duerrschmid, C., Bournat, J., Constable, P., Jain, M., Xia, F., Saha, P. K., Del Solar, M., Zhu, B., York, B., Sarkar, P., Rendon, D. A., Gaber, M. W., LeMaire, S. A., Coselli, J. S., Milewicz, D. M., Sutton, V. R., Butte, N. F., Moore, D. D., and Chopra, A. R. (2016) Asprosin, a Fasting-Induced Glucogenic Protein Hormone. Cell 165, 566-579

8. Lonnqvist, L., Reinhardt, D., Sakai, L., and Peltonen, L. (1998) Evidence for furin-type activity-mediated C-terminal processing of profibrillin- 1 and interference in the processing by certain mutations. Hum Mol Genet 7, 2039-2044

9. $\quad$ Li, E., Shan, H., Chen, L., Long, A., Zhang, Y., Liu, Y., Jia, L., Wei, F., Han, J., Li, T., Liu, X., Deng, H., and Wang, Y. (2019) OLFR734 Mediates Glucose Metabolism as a Receptor of Asprosin. Cell Metab 30, 319-328.e318

10. Lee, T., Yun, S., Jeong, J. H., and Jung, T. W. (2019) Asprosin impairs insulin secretion in response to glucose and viability through TLR4/JNK-mediated inflammation. Mol Cell Endocrinol 486, 96-104

11. Duerrschmid, C., He, Y., Wang, C., Li, C., Bournat, J. C., Romere, C., Saha, P. K., Lee, M. E., Phillips, K. J., Jain, M., Jia, P., Zhao, Z., Farias, M., Wu, Q., Milewicz, D. M., Sutton, V. R., Moore, D. D., Butte, N. F., Krashes, M. J., Xu, Y., and Chopra, A. R. (2017) Asprosin is a centrally acting orexigenic hormone. Nat Med 23, 1444-1453

12. Jung, T. W., Kim, H. C., Kim, H. U., Park, T., Park, J., Kim, U., Kim, M. K., and Jeong, J. H. (2019) Asprosin attenuates insulin signaling pathway through PKC $\delta$-activated ER stress and inflammation in skeletal muscle. J Cell Physiol 234, 20888-20899

13. Hoffmann, J. G., Xie, W., and Chopra, A. R. (2020) Energy Regulation Mechanism and Therapeutic Potential of Asprosin. Diabetes 69, 559-566 
14. Morcos, Y. A. T., Lütke, S., Tenbieg, A., Hanisch, F.G., Pryymachuk, G., Piekarek, N., Hoffmann, T., Keller, T., Janoschek, R., Niehoff, A., Zaucke, F., Dötsch, J., HucklenbruchRother, E., Sengle, G. (2021) Sensitive asprosin detection in clinical samples reveals serum/ saliva correlation and indicates cartilage as source for serum asprosin. Sci Rep, in press

15. Qian, R. Q., and Glanville, R. W. (1997) Alignment of fibrillin molecules in elastic microfibrils is defined by transglutaminase-derived cross-links. Biochemistry 36, 15841-15847

16. Coussons, P. J., Price, N. C., Kelly, S. M., Smith, B., and Sawyer, L. (1992) Factors that govern the specificity of transglutaminase-catalysed modification of proteins and peptides. Biochem J 282 ( Pt 3), 929-930

17. Eckert, R. L., Kaartinen, M. T., Nurminskaya, M., Belkin, A. M., Colak, G., Johnson, G. V., and Mehta, K. (2014) Transglutaminase regulation of cell function. Physiol Rev 94, 383-417

18. Yu, Y., He, J. H., Hu, L. L., Jiang, L. L., Fang, L., Yao, G. D., Wang, S. J., Yang, Q., Guo, Y., Liu, L., Shang, T., Sato, Y., Kawamura, K., Hsueh, A. J., and Sun, Y. P. (2020) Placensin is a glucogenic hormone secreted by human placenta. EMBO Rep 21, e49530

19. Turner, P. M., and Lorand, L. (1989) Complexation of fibronectin with tissue transglutaminase. Biochemistry 28, 628-635

20. Akimov, S. S., and Belkin, A. M. (2001) Cell-surface transglutaminase promotes fibronectin assembly via interaction with the gelatin-binding domain of fibronectin: a role in TGFbetadependent matrix deposition. J Cell Sci 114, 2989-3000

21. Jung, T. W., Kim, H. C., Kim, H. U., Park, T., Park, J., Kim, U., Kim, M. K., and Jeong, J. H. (2019) Asprosin attenuates insulin signaling pathway through PKCdelta-activated ER stress and inflammation in skeletal muscle. J Cell Physiol 234, 20888-20899

22. Zhang, Y., Zhu, Z., Zhai, W., Bi, Y., Yin, Y., and Zhang, W. (2021) Expression and purification of asprosin in Pichia pastoris and investigation of its increase glucose uptake activity in skeletal muscle through activation of AMPK. Enzyme Microb Technol 144, 109737

23. Clarke, A. W., Wise, S. G., Cain, S. A., Kielty, C. M., and Weiss, A. S. (2005) Coacervation is promoted by molecular interactions between the PF2 segment of fibrillin-1 and the domain 4 region of tropoelastin. Biochemistry 44, 10271-10281

24. Rock, M. J., Cain, S. A., Freeman, L. J., Morgan, A., Mellody, K., Marson, A., Shuttleworth, C. A., Weiss, A. S., and Kielty, C. M. (2004) Molecular basis of elastic fiber formation. Critical interactions and a tropoelastin-fibrillin-1 cross-link. J Biol Chem 279, 23748-23758

25. Zhong, L., Long, Y., Wang, S., Lian, R., Deng, L., Ye, Z., Wang, Z., and Liu, B. (2020) Continuous elevation of plasma asprosin in pregnant women complicated with gestational diabetes mellitus: A nested case-control study. Placenta 93, 17-22

26. Rossin, F., D'Eletto, M., Falasca, L., Sepe, S., Cocco, S., Fimia, G. M., Campanella, M., Mastroberardino, P. G., Farrace, M. G., and Piacentini, M. (2015) Transglutaminase 2 ablation leads to mitophagy impairment associated with a metabolic shift towards aerobic glycolysis. Cell Death Differ 22, 408-418

27. Zimmermann, L. A., Correns, A., Furlan, A. G., Spanou, C. E. S., and Sengle, G. (2021) Controlling BMP growth factor bioavailability: The extracellular matrix as multi skilled platform. Cell Signal 85, 110071

28. Sengle, G., and Sakai, L. Y. (2015) The fibrillin microfibril scaffold: A niche for growth factors and mechanosensation? Matrix Biol 47, 3-12

29. Isogai, Z., Ono, R. N., Ushiro, S., Keene, D. R., Chen, Y., Mazzieri, R., Charbonneau, N. L., Reinhardt, D. P., Rifkin, D. B., and Sakai, L. Y. (2003) Latent transforming growth factor beta-binding protein 1 interacts with fibrillin and is a microfibril-associated protein. $J$ Biol Chem 278, 2750-2757

30. Sengle, G., Charbonneau, N. L., Ono, R. N., Sasaki, T., Alvarez, J., Keene, D. R., Bachinger, H. P., and Sakai, L. Y. (2008) Targeting of bone morphogenetic protein growth factor complexes to fibrillin. J Biol Chem 283, 13874-13888

31. Sengle, G., Ono, R. N., Sasaki, T., and Sakai, L. Y. (2011) Prodomains of transforming growth factor beta (TGFbeta) superfamily members specify different functions: extracellular matrix interactions and growth factor bioavailability. J Biol Chem 286, 5087-5099

32. Reinhardt, D. P., Keene, D. R., Corson, G. M., Poschl, E., Bachinger, H. P., Gambee, J. E., and Sakai, L. Y. (1996) Fibrillin-1: organization in microfibrils and structural properties. $J$ Mol Biol 258, 104-116 
33. Aeschlimann, D., and Paulsson, M. (1991) Cross-linking of laminin-nidogen complexes by tissue transglutaminase. A novel mechanism for basement membrane stabilization. J Biol Chem 266, 15308-15317

\section{Figure legends}

\section{Figure 1: Tissue staining reveals presence of asprosin positive fibers}

Confocal immunofluorescence microscopy of asprosin in human tissues. Sections were fixed with acetone and incubated with pc-asp (green) and DAPI (blue). Endogenous asprosin signals were detected extra- and intracellular and mainly in two staining patterns: (top panel, white arrow) fiber-like structure in placenta and cardiac muscle, and (bottom, white arrow heads) diffused versicle-like structure in liver. Images were obtained from a Leica SP8 confocal microscope and were processed using Leica LAS AF Lite 4.0 software and Fiji/ImageJ software to obtain average intensity Zprojection.

\section{Figure 2: Asprosin oligomers and fibers detected in cell culture}

A. Immunoblot analysis of asprosin produced by cells in cell lysates and cell culture supernatants. Asprosin was detected as a band at the corresponding molecular weight of $35 \mathrm{kDa}$ and asprosin positive bands of higher molecular weight in both cell lysates and supernatants. B. Immunofluorescence analysis of asprosin after 7-9 days of culturing in 3T3-L1, primary human dermal fibroblasts (HDF), and primary human chondrocytes (HCH). 3T3-L1 cells were incubated with mab anti-asprosin (anti-mouse) and HDF, and $\mathrm{HCH}$ with pc-asp (anti-rabbit) (green) and DAPI (blue). The staining revealed depositions of asprosin positive fibers as indicated by white arrows. Images were obtained from Axiophot Microscope (Carl Zeiss, Germany) and Leica SP8 confocal microscope and were processed using Leica LAS AF Lite 4.0 software and Fiji/ImageJ software to obtain average intensity Z-projection.

\section{Figure 3: Oligomerization and fiber formation upon overexpression of monomeric asprosin}

A. Immunoblotting of cell lysates and cell culture supernatants from HEK-293 and 3T3-L1 transfected with pCEP-Pu Vector overexpressing asprosin-2×Strep-tag II using StrepMAB-Classic antibody (anti- 
mouse). The immunoblots revealed the secretion of monomeric asprosin as observed in HEK-293, and positive asprosin bands with high molecular weight in HEK-293 cell lysate fraction, and mostly detected in lysate and supernatant of 3T3-L1 cells. B. Immunostaining of the asprosin-transfected HEK-293 and 3T3-L1 cells with StrepMAB-Classic antibody showing asprosin intra- and extracellular positive signals. The extracellular signals reveal deposition of asprosin positive fibers as indicated by white arrows. Images were obtained from Axiophot Microscope (Carl Zeiss, Germany) and Leica SP8 confocal microscope and were processed using Leica LAS AF Lite 4.0 software and Fiji/ImageJ software to obtain average intensity Z-projection.

\section{Figure 4: In-vitro asprosin crosslinking by transglutaminase 2 (TG2)}

A. Incubation of monomeric recombinant asprosin with TG2 results in a significant shift of asprosin positive immunoblot signals towards higher molecular weight positions. B. Incubation of monomeric recombinant asprosin with a chemical crosslinker, DSS showed the same higher molecular weight bands as after incubation with TG2. C. The crosslinking reaction between asprosin and monodansylcadaverine (MDC), TG2 substrate and a dye detectable by UV light. D. Incubation of monomeric recombinant asprosin with monodansylcadaverine (MDC) in the presence of TG2, results in bands corresponding to monomeric and dimeric asprosin detected by UV light. The band marked by dashed white box was analysed by mass spectrometery to identify the poteintal glutamine residues subjected to TG2 modification. E. Mass spectrometry analysis revealed the glutamine residues which could be utilized by TG2 for asprosin (marked in red, position regarding the fibrillin-1 sequence in parentheses). Underlined residues mark predicted cleavage sites of Lys-C and trypsin used to generate peptides of MDC crosslinked asprosin. Pc-asp (anti-rabbit) was used in the presented immunoblots.

\section{Figure 5: Assembly and ECM deposition of administered recombinant asprosin}

Recombinant asprosin $(5 \mu \mathrm{g} / \mathrm{ml})$ was administered to cell cultures of U2-OS, RPE, and HDF cells. $48 \mathrm{~h}$ after asprosin treatment, cells were immunostained with anti-fibronectin (green), pc-asp (red), and DAPI (blue). Immunofluorescence analysis revealed the presence of an intact fibronectin fiber network in cultures of RPE and HDF cells, but not in cultures of U2-OS cells. Asprosin 
immunostaining showed that asprosin is detected as aggregates in asprosin-treated U2-OS cells. Asprosin positive fibers were observed after administration to RPE cells or HDF. Images were obtained from a Leica SP8 confocal microscope and were processed using Leica LAS AF Lite 4.0 software and Fiji/ImageJ software to obtain average intensity Z-projection. Scale bar: $50 \mu \mathrm{m}$.

\section{Figure 6: Asprosin interacts with the N-terminal region of fibrillin-1}

A. Domain structure of fibrillin-1 and its N- and C-terminal halves including the start-EGF4 fragment (furin cleavage site marked by arrow). B. EBNA cells transfected with overexpression constructs encoding for fibrillin-1 N- and C-terminal halves (rF90 and rF6). Medium obtained from nontransfected EBNA cells served as control (ctrl). Media obtained from the transfected with rF90 and rF6 are indicated. EBNA control medium supplemented with fibronectin $(10 \mu \mathrm{g})$ is indicated as FN. The media were subjected to incubation with recombinant asprosin $(2 \times$ strep tagged asprosin) overnight at $4^{\circ} \mathrm{C}$. The mixtures were then subjected to pulldown with Strep-Tactin XT beads. After washing, the eluted fractions were subjected to immunoblot analysis with the indicated antibodies. $\mathbf{C}$. SPR binding studies suggesting that asprosin interacts with the N-terminal half of fibrillin-1 (rF90) and the start-EGF4 fragment, but does not bind to fibronectin.

\section{Figure 7: Linear deposition of asprosin into fibers is fibrillin-1 dependent}

A. The formation of asprosin positive fibers after administration of recombinant asprosin $(5 \mu \mathrm{g} / \mathrm{ml})$ depend on the integrity of the fibrillin fibril network which is deficient in primary fibroblast cultures from MFS patients. Control and MFS patients' cells were incubated with anti-fibronectin (anti-mouse, cyan), anti-fibrillin-1 (rF90) (anti-rabbit, green), murine-asprosin antibody (anti-rabbit, red), and DAPI (white). B. Quantification of the mean asprosin fluorescence intensity in MFS patients' cells normalized to fluorescence intensity of control HDF. Images were obtained from a Leica SP8 confocal microscope and were processed using Leica LAS AF Lite 4.0 software and Fiji/ImageJ software to obtain average intensity Z-projection. 


\section{Figure 8: Asprosin and fibrillin-1 are colocalizing in murine tissues}

Confocal immunofluorescence microscopy of asprosin in indicated mouse tissues. Sections were fixed with acetone and incubated with murine-asprosin antibody (red), anti-fibrillin-1(cyan) and DAPI (blue). The immunostaining revealed depositions of asprosin positive fibers, which are co-localizing with fibrillin-1 positive fibers. Images were obtained from an Olympus BX43 microscope (Olympus) equipped with DP80 dual CCD camera. ImageJ software was used for image processing.

\section{Figure 9: Mechanistic model for targeting of asprosin to the extracellular matrix}

The model is showing a potential mechanism of asprosin storage and utilization within the extracellular matrix. Upon secretion of asprosin may be targeted to assebled fibrillin fibers. Thereby, TG2 mediated crosslinking enables stable storage of asprosin in the form of oligomers. Oligomeric asprosin may be later utilitzed by specific activation mechanisms such as proteolytic degradation of asprosin positive fibers. Released asprosin may act locally or is transported via the circulation to other target organs. 


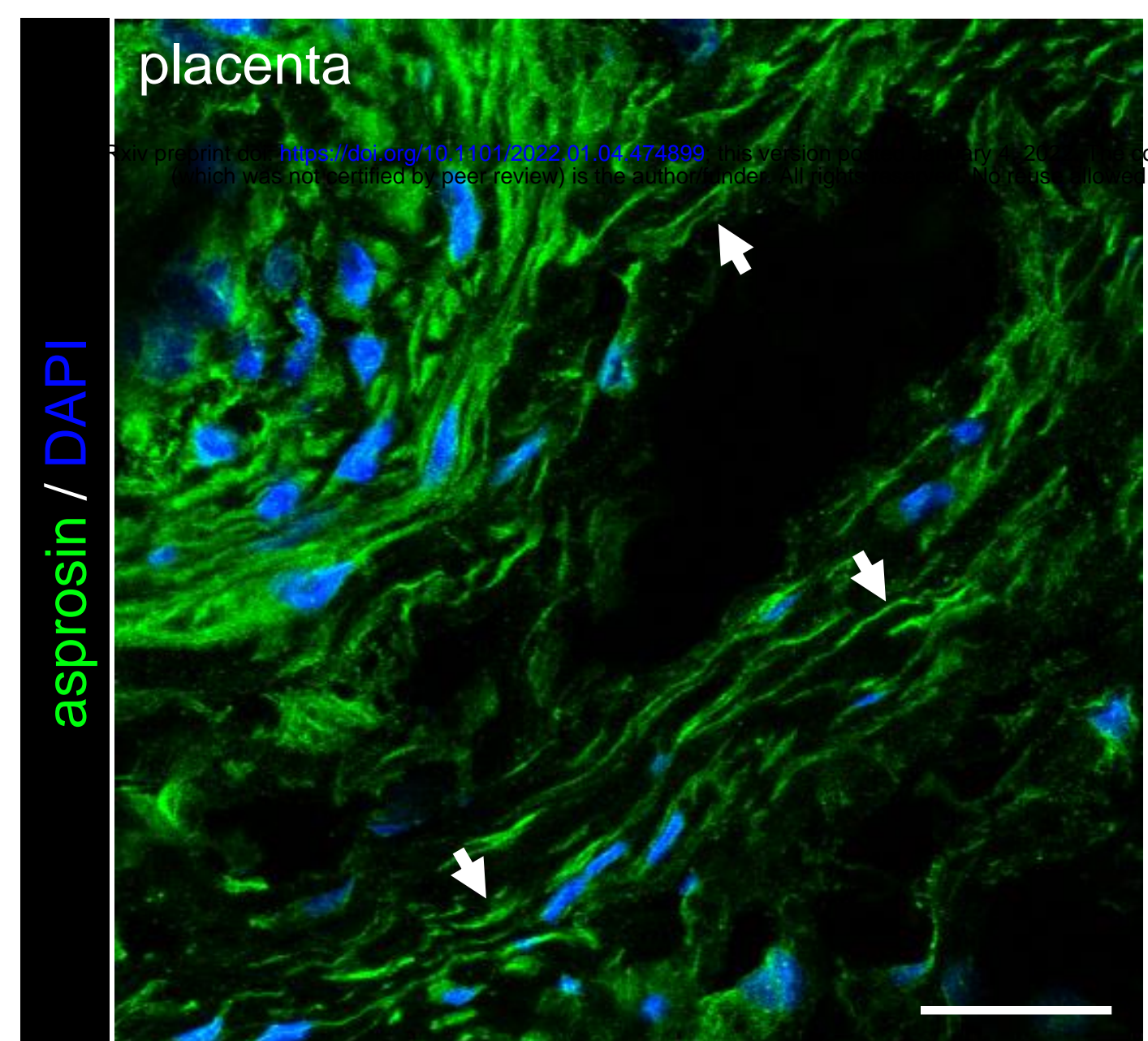

cardiac muscle

$-4 x^{2}=$

cardiac

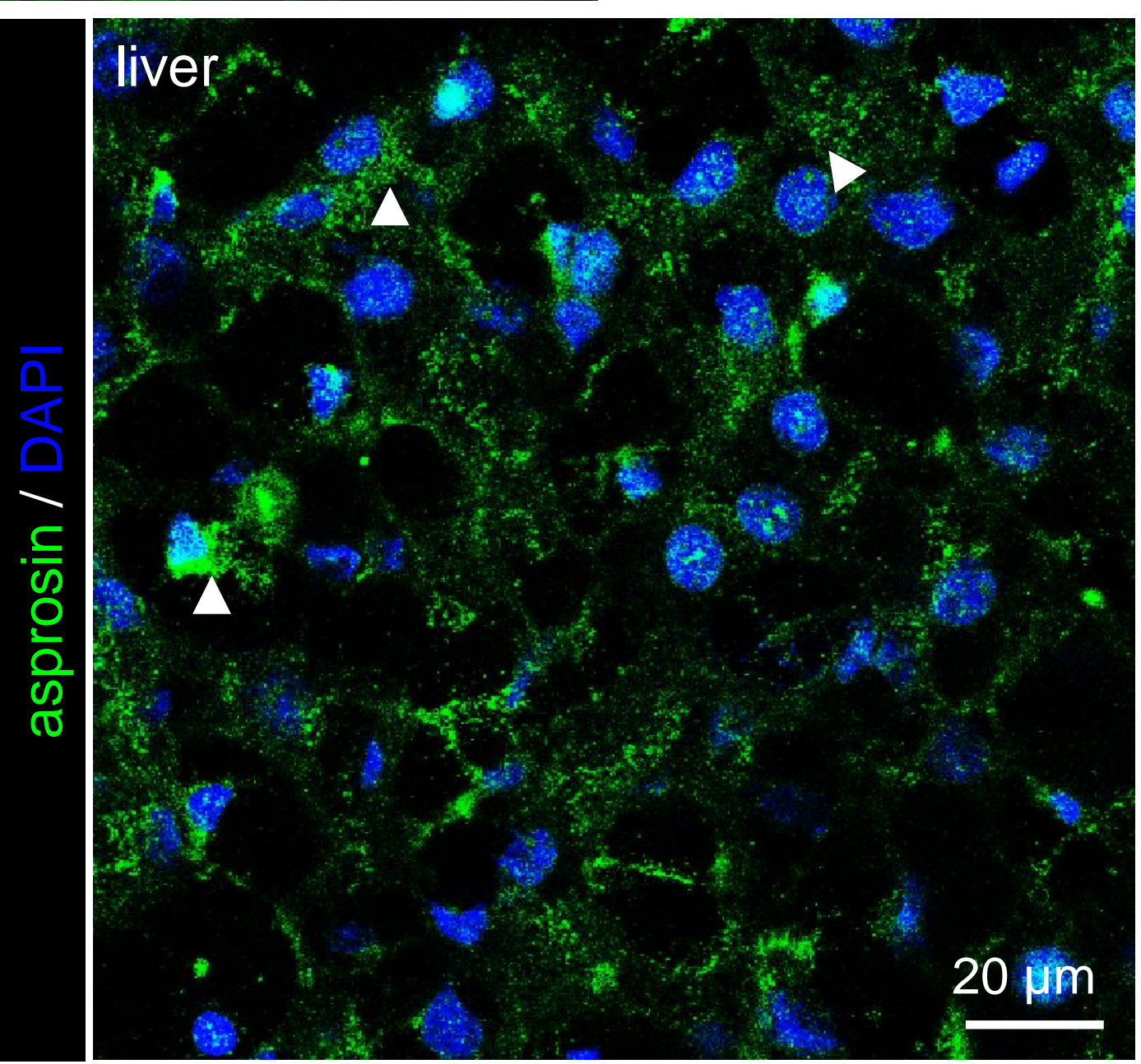

Figure 1 


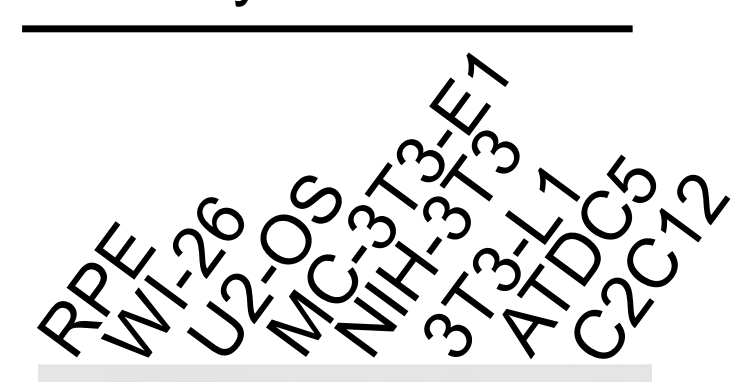
$\mathrm{kDa}$
$\mathrm{kDa}$
$\frac{2}{\overline{0}} \mid \begin{aligned} & 250-\bar{E} \\ & 130-\end{aligned}$
250-
$130-$
Oे
$\frac{0}{0}$
$\frac{1}{1}$
$\frac{1}{\frac{1}{\sigma}}$
$100-$
$50-$
$0=-\div=$
$100-$
$70-$
55-
35-
25-
$35-$
$25-$

3T3-L1

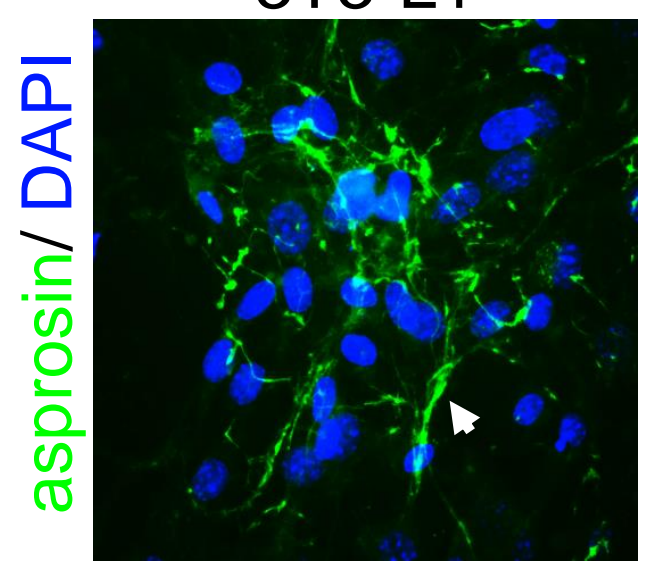

HDF

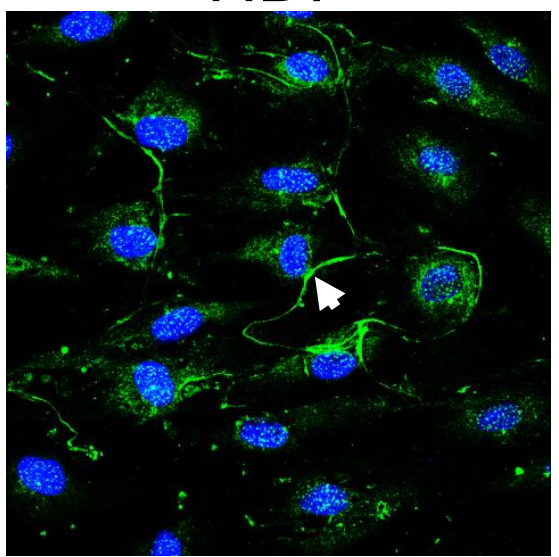

$\mathrm{HCH}$

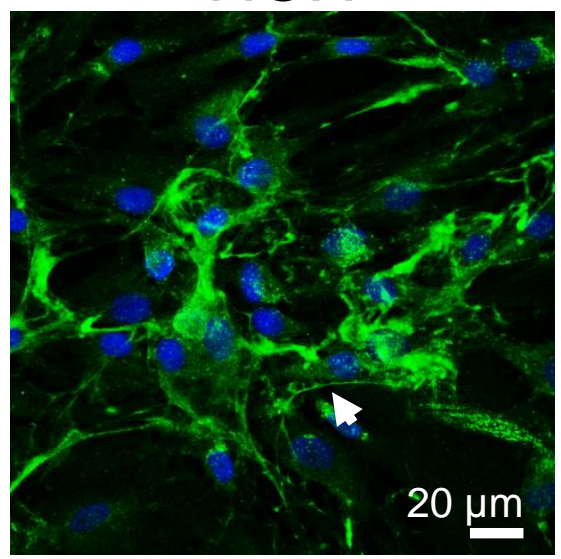

Figure 2 
A

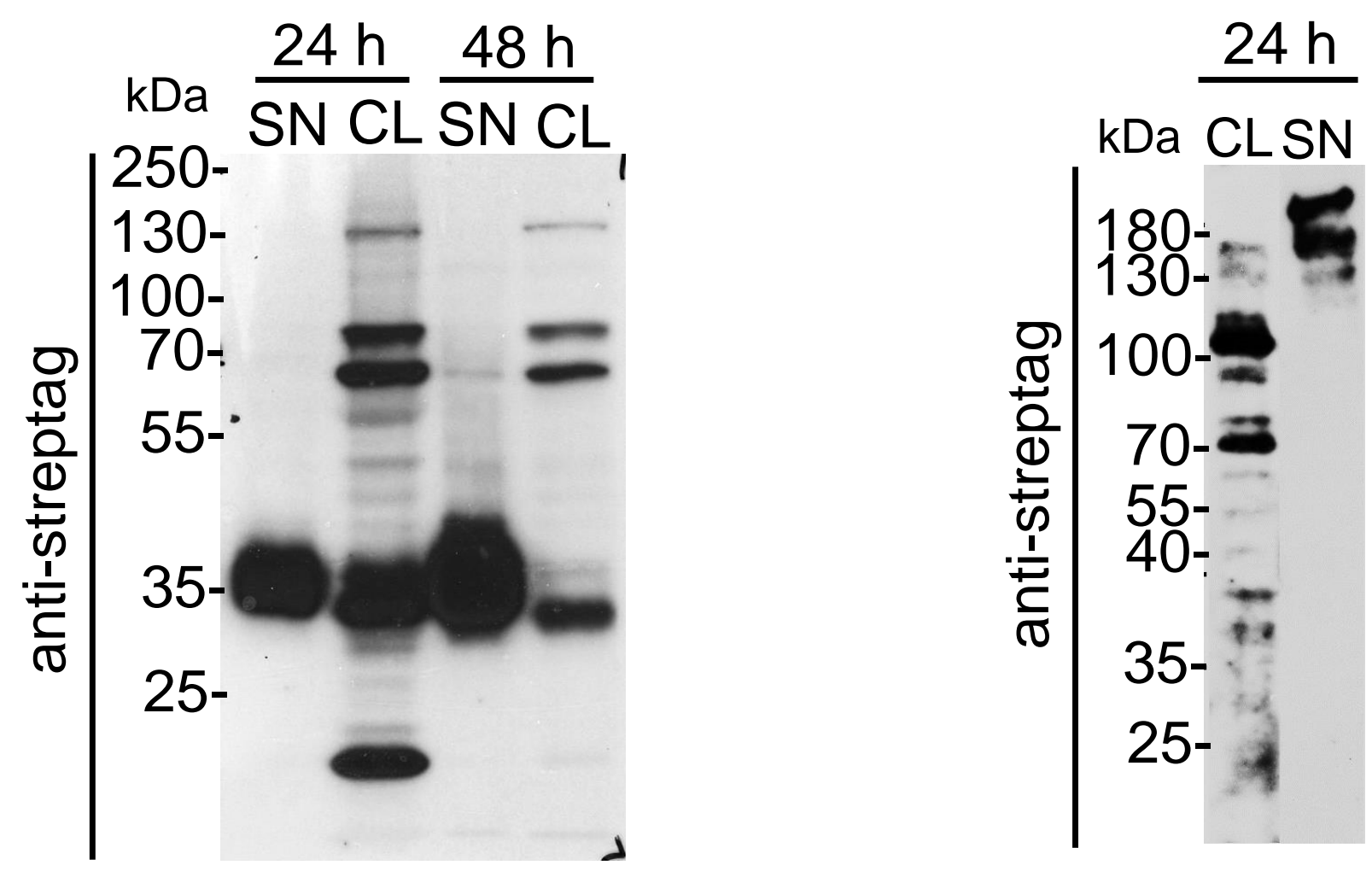

B

transfected asprosin

HEK 293-asprosin

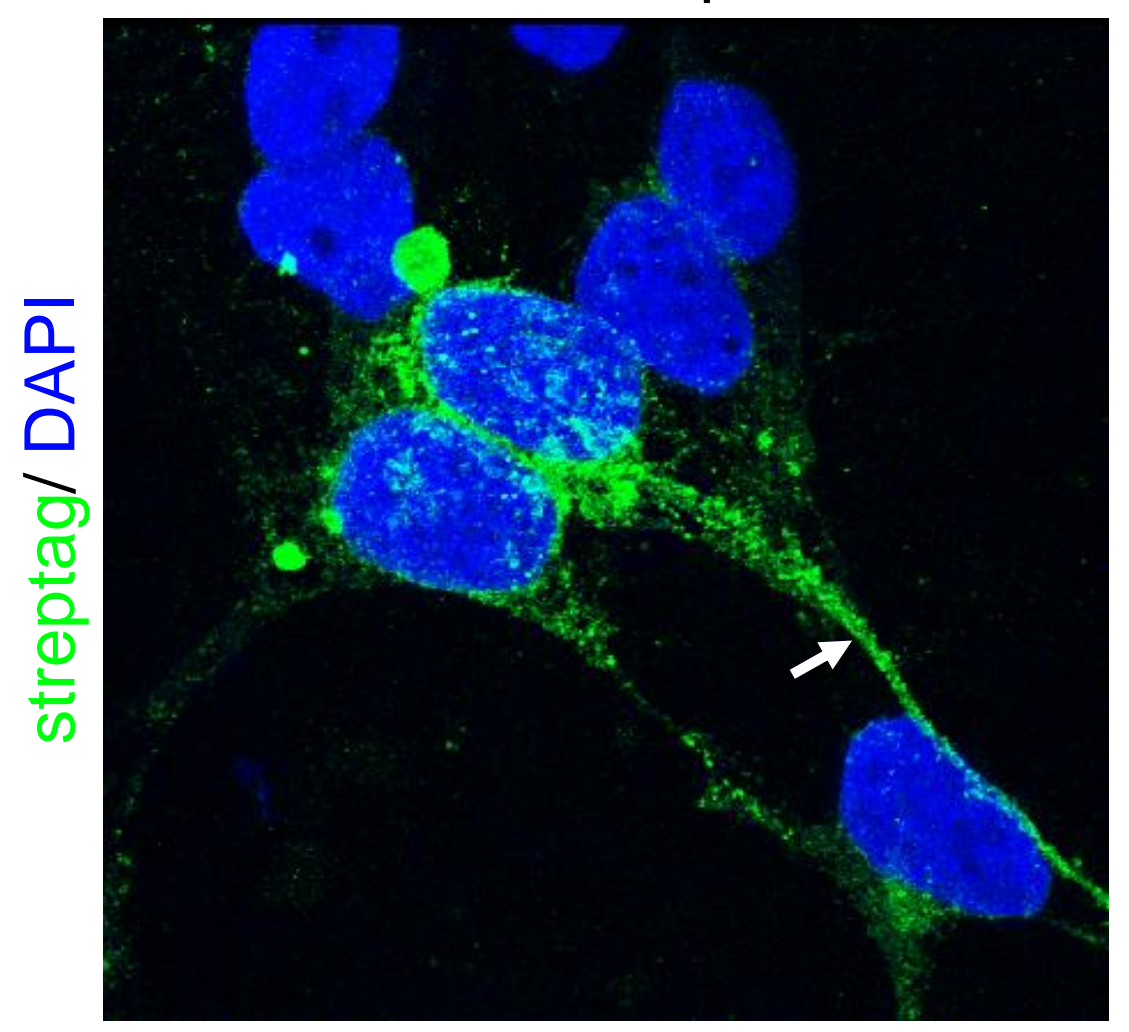

3t3-L1-asprosin

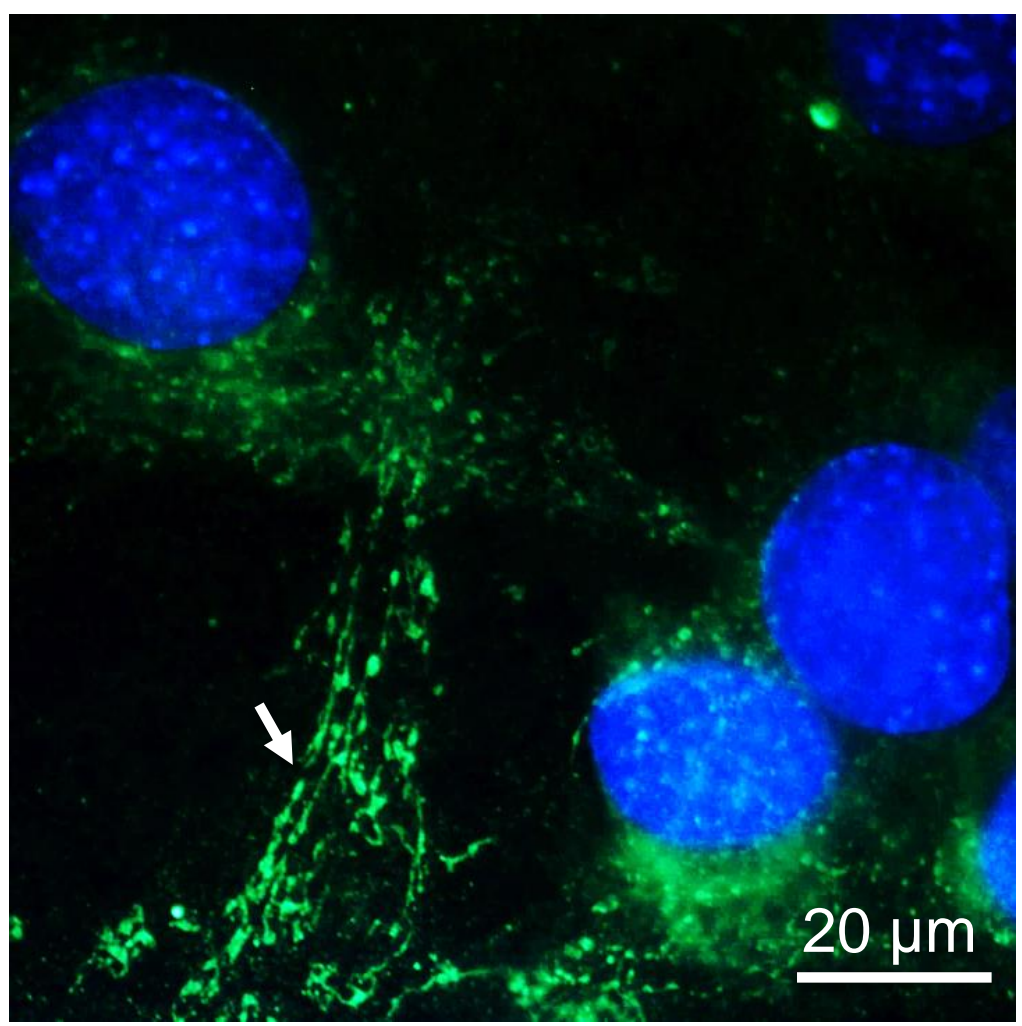


A

TG2 (min) $0 \quad 51015 \quad$ DSS $(\mu \mathrm{M}) \quad 010204080$

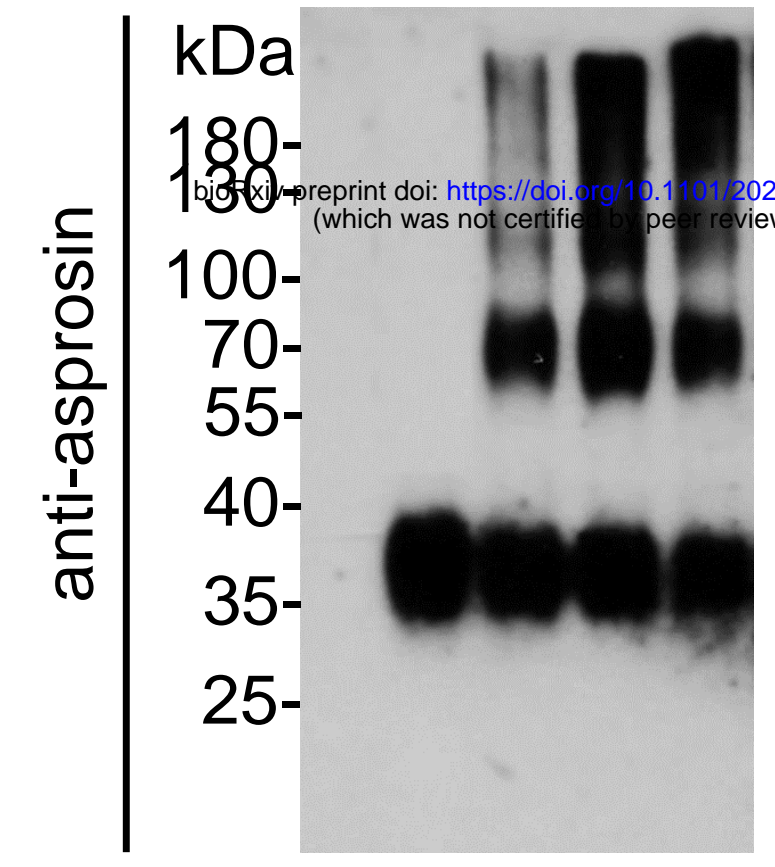
$\mathrm{kDa} 110 \mathrm{a}$ asprosin 180-- 8 - monodansylcadaverine (MDC) / MW: $335.46 \mathrm{Da}$
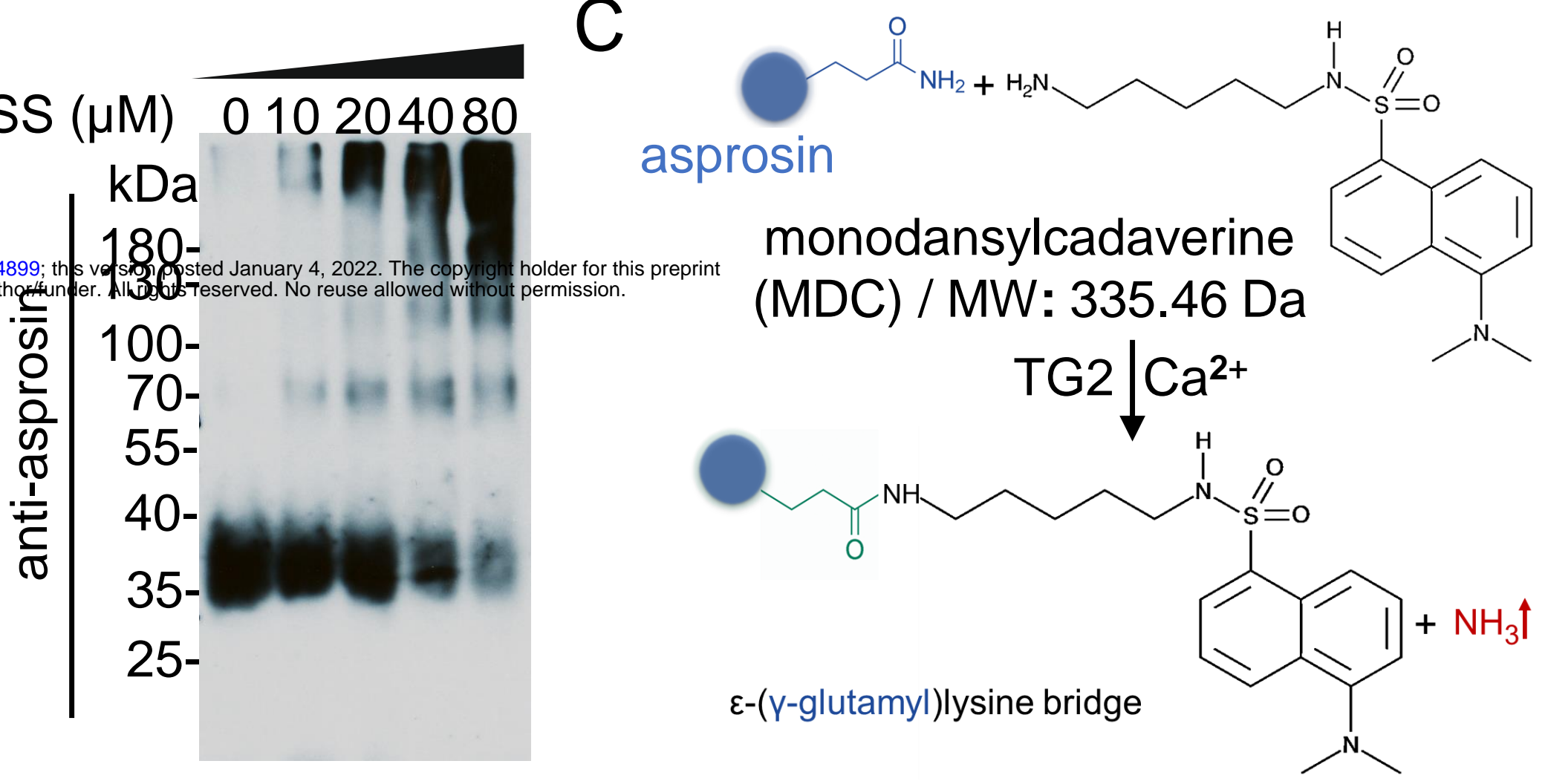

$\mathrm{D}$

TG2 (min) $0 \quad 5 \quad 10 \quad 1520 \quad 3045$

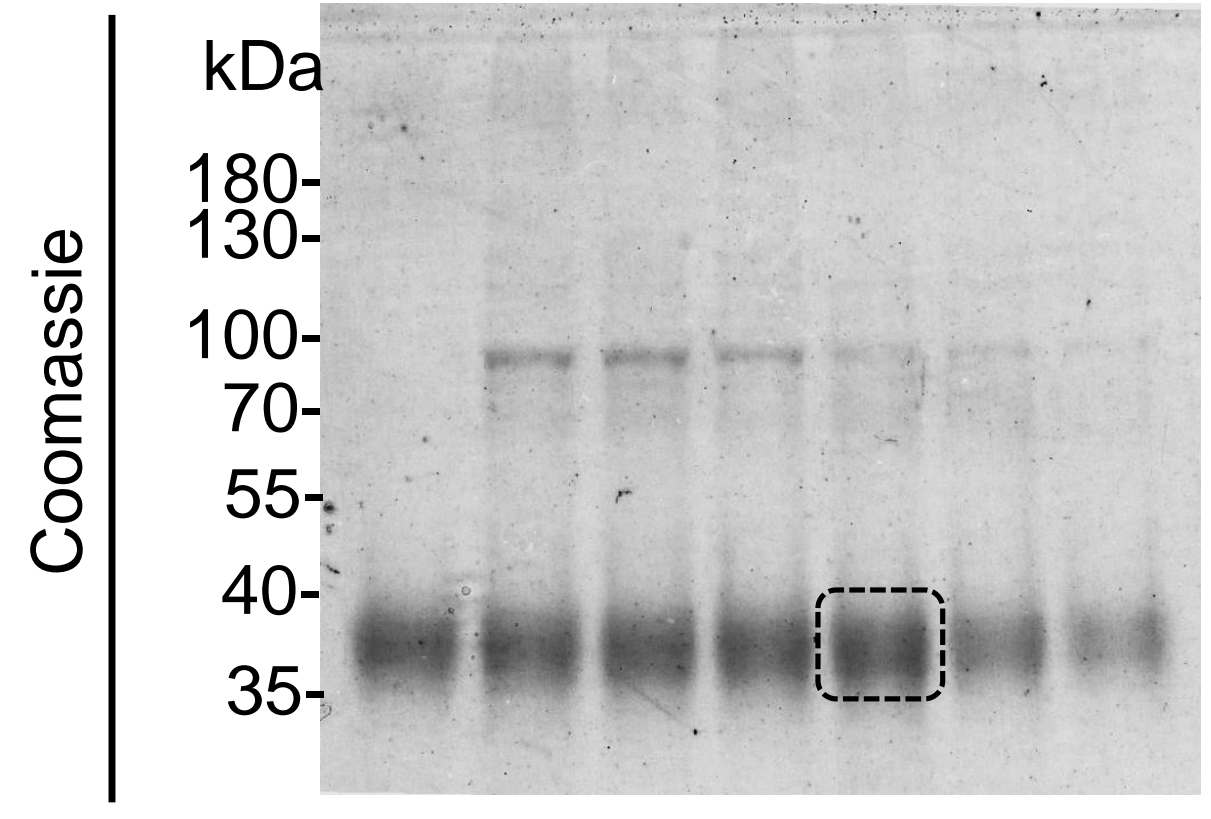

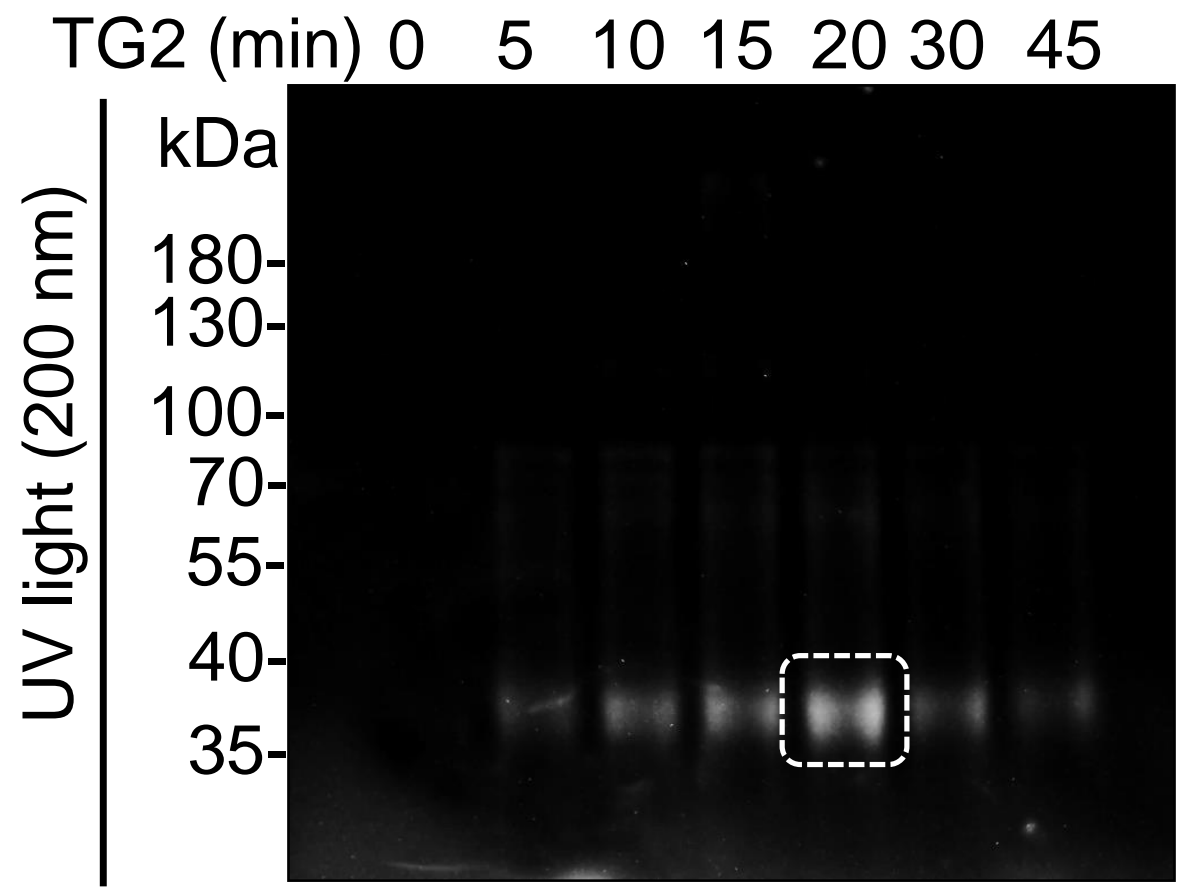

E

STNETDASNI EDQSETEANV SLASWDVEKT AIFAFNISHV SNKVRILELL PALTTLTNHN

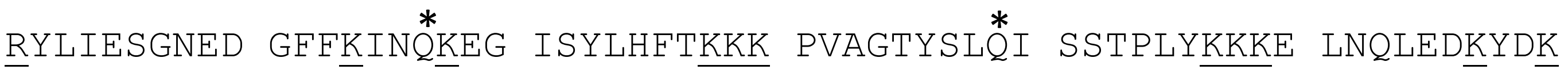
DYLSGELGDN LKMKIQVLLH GSLVPRGSAW SHPQFEKGGG SGGGSGGGSW SHPQFEK Strep-tag II Strep-tag II 


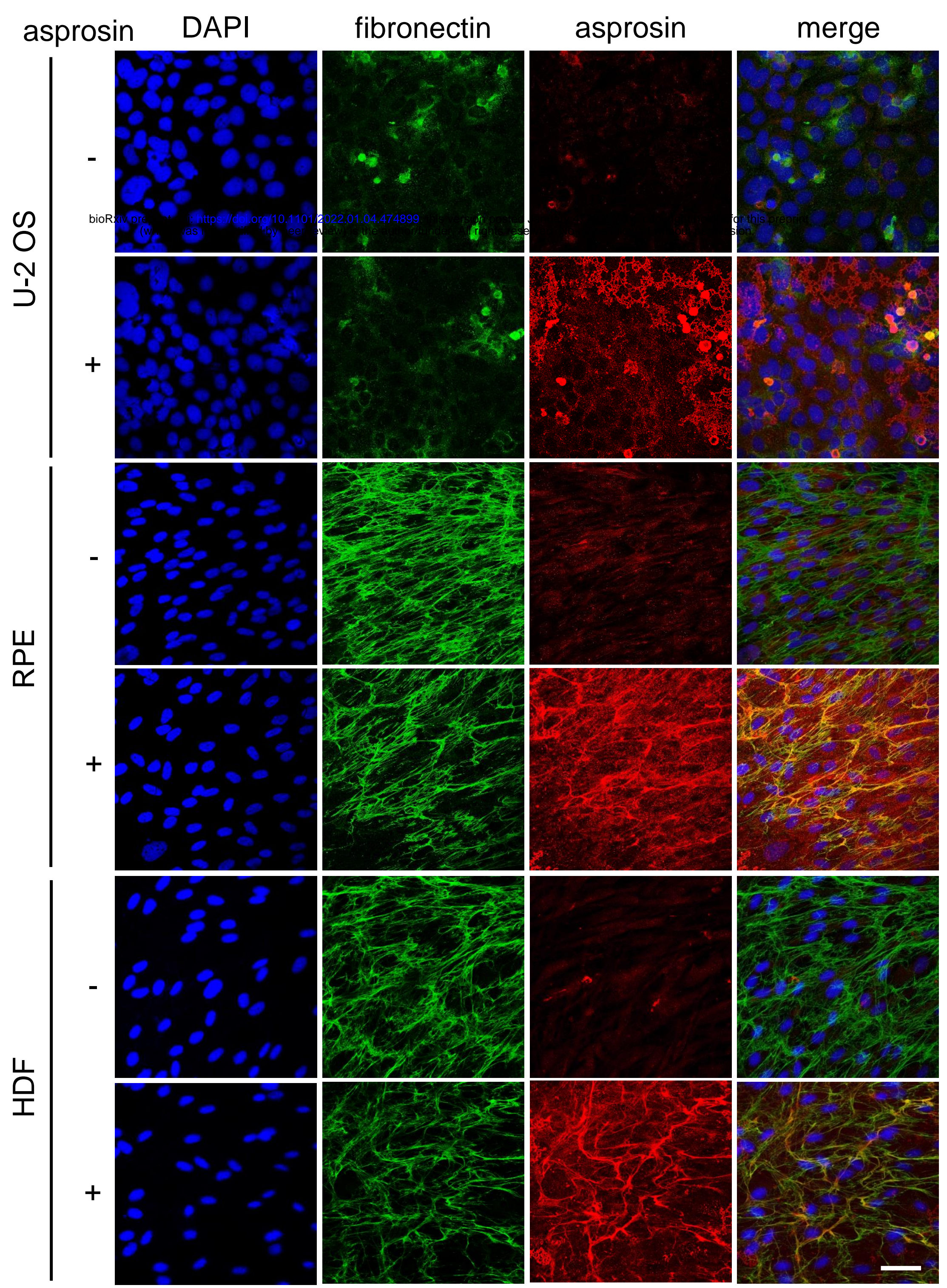

Figure 5 
bioRxiv preprint doi: https://doi.org/10.1101/2022.01.04.474899; this version posted January 4, 2022. The copyright holder for this preprint
(which was not certified by peer review) is the author/funder. All rights reserved. No reuse allowed without permfspipkr.In

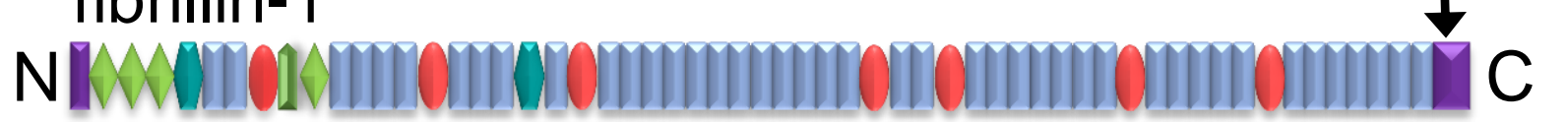

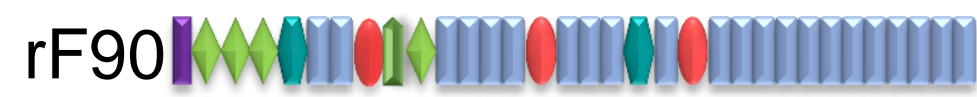
start-EGF4

B

C
Iunique region EFG-like domain asprosin

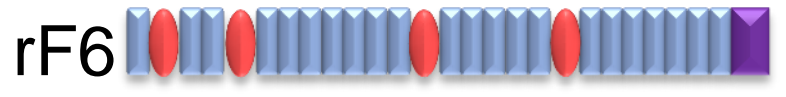

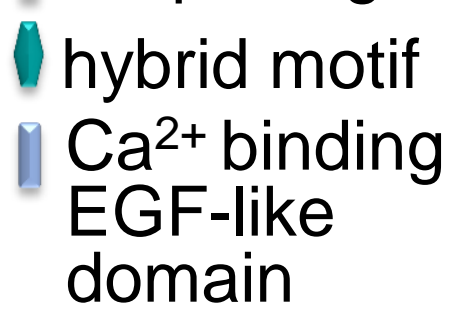

I proline rich region

$\mathrm{Ca}^{2+}$ binding 8-cysteine domain EGF-like domain

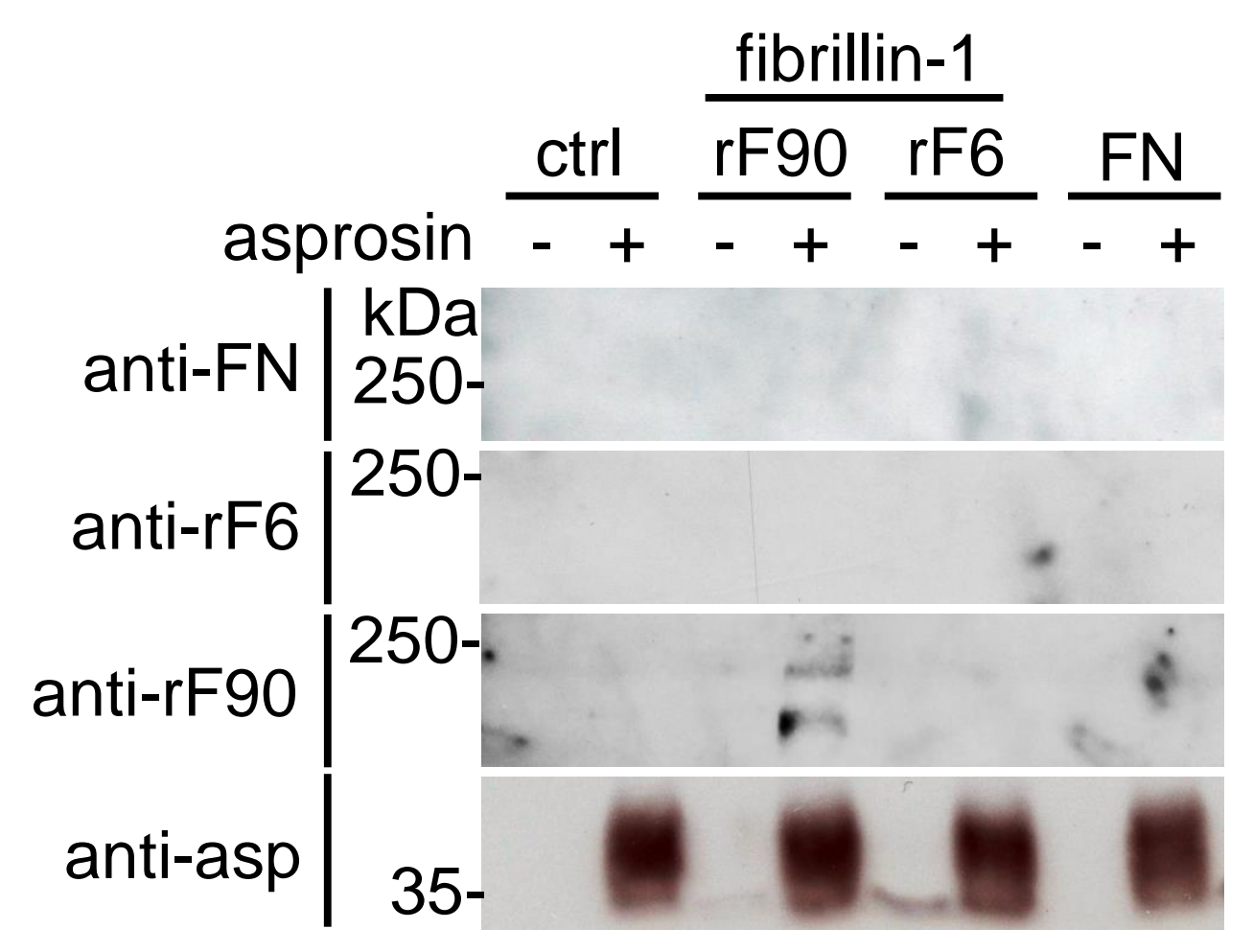

0 - 320 nM rF90 injected onto asprosin on chip

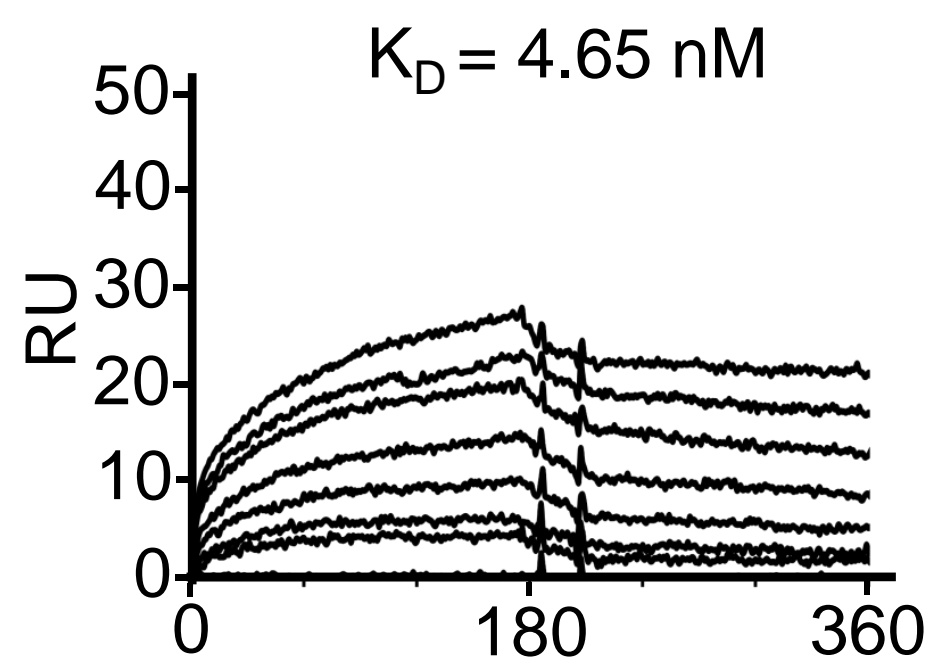

0 - 320 nM start-EGF4 injected onto asprosin on chip

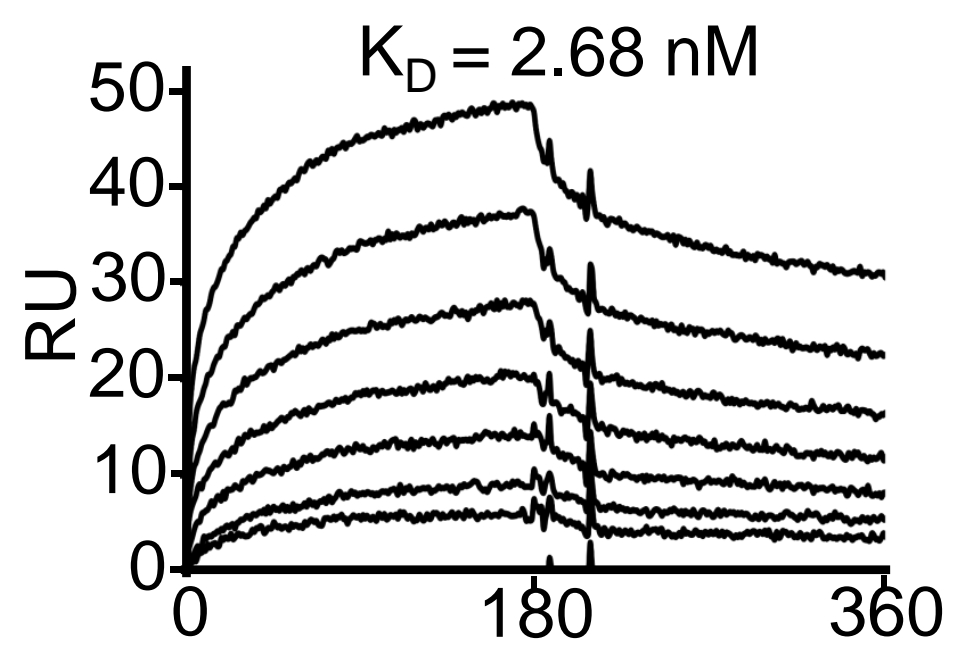

0 - $320 \mathrm{nM}$ fibronectin injected onto asprosin on chip

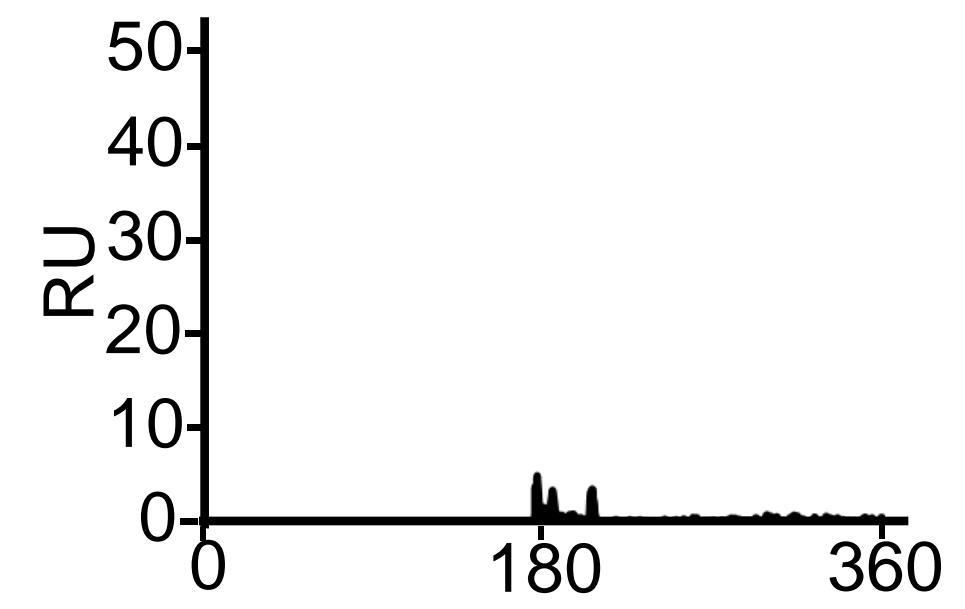

Figure 6 


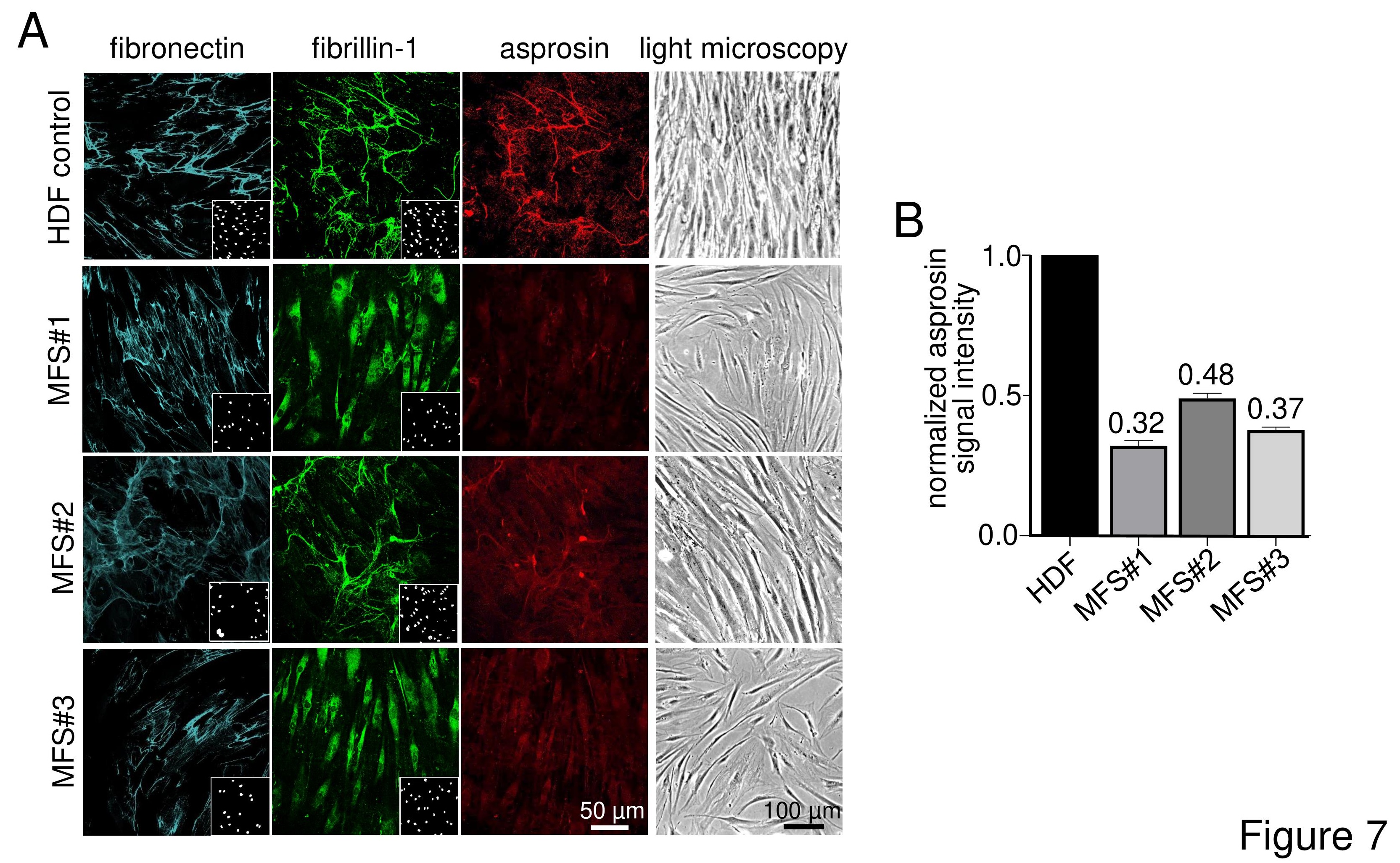




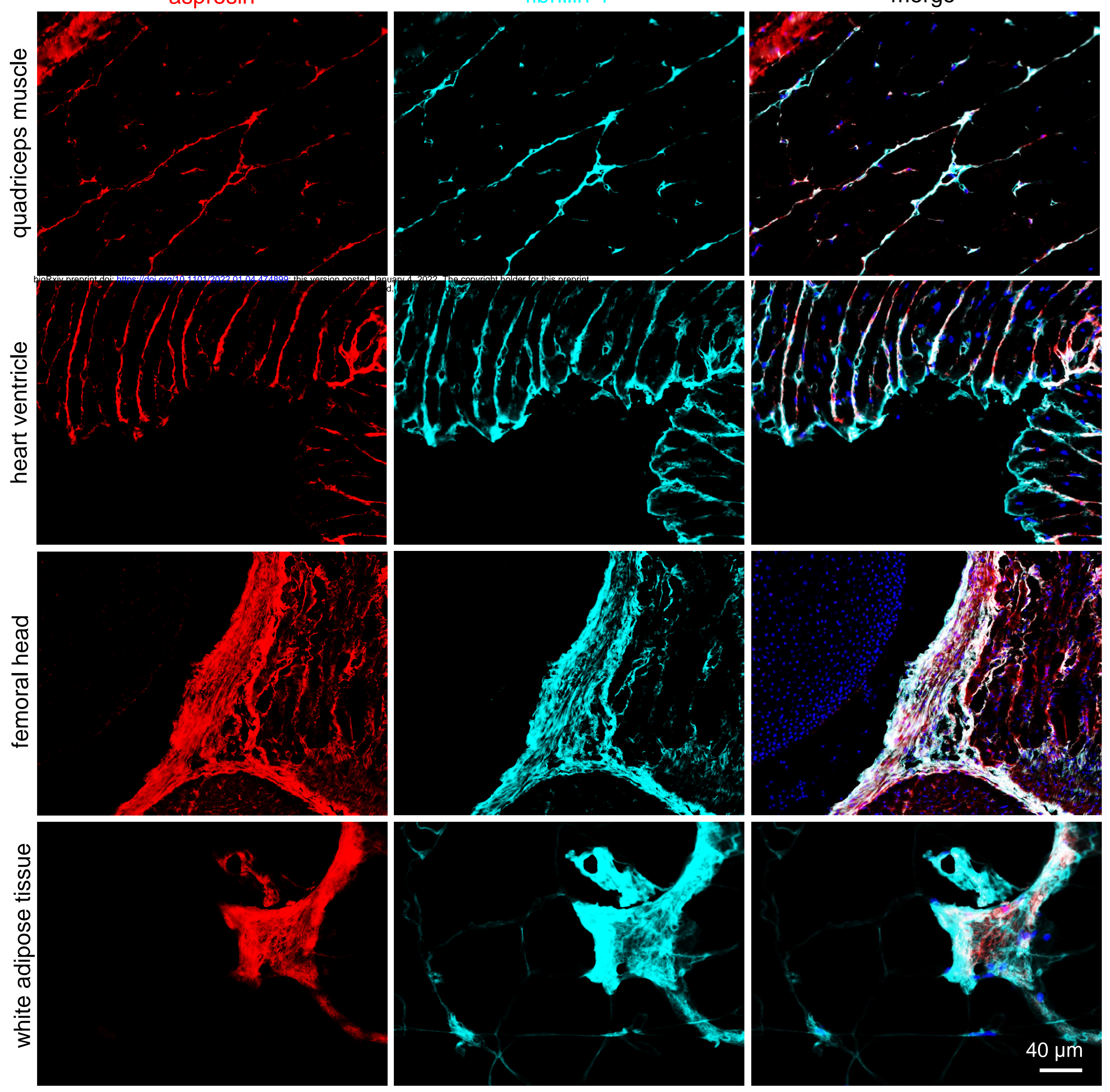

Figure 8 
extracellular

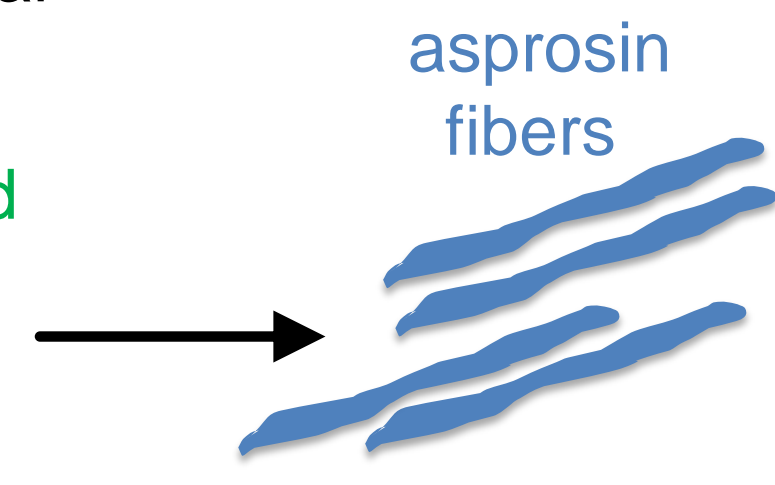

fibrillin-1 microfibril scaffold

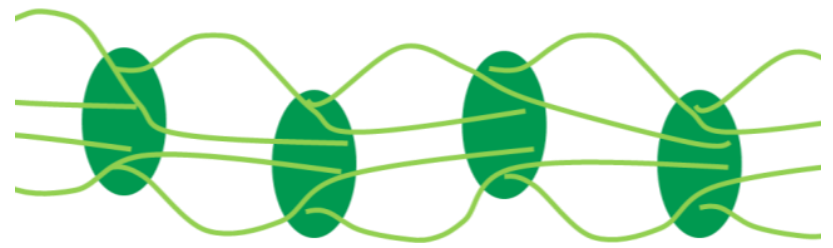

\section{circulation}

$?$

local energy demand, ECM changes

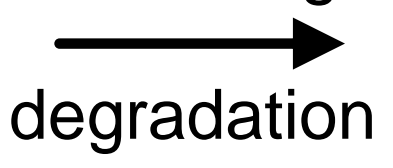

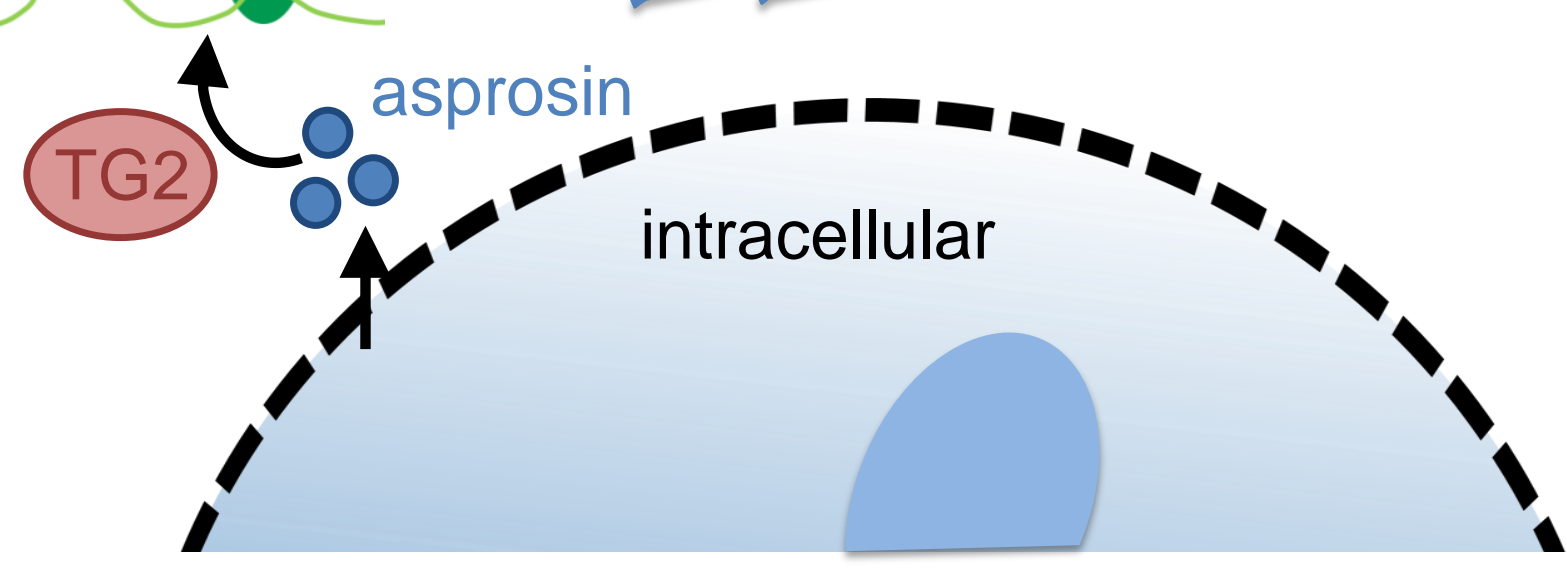

Figure 9 


\section{$A$}
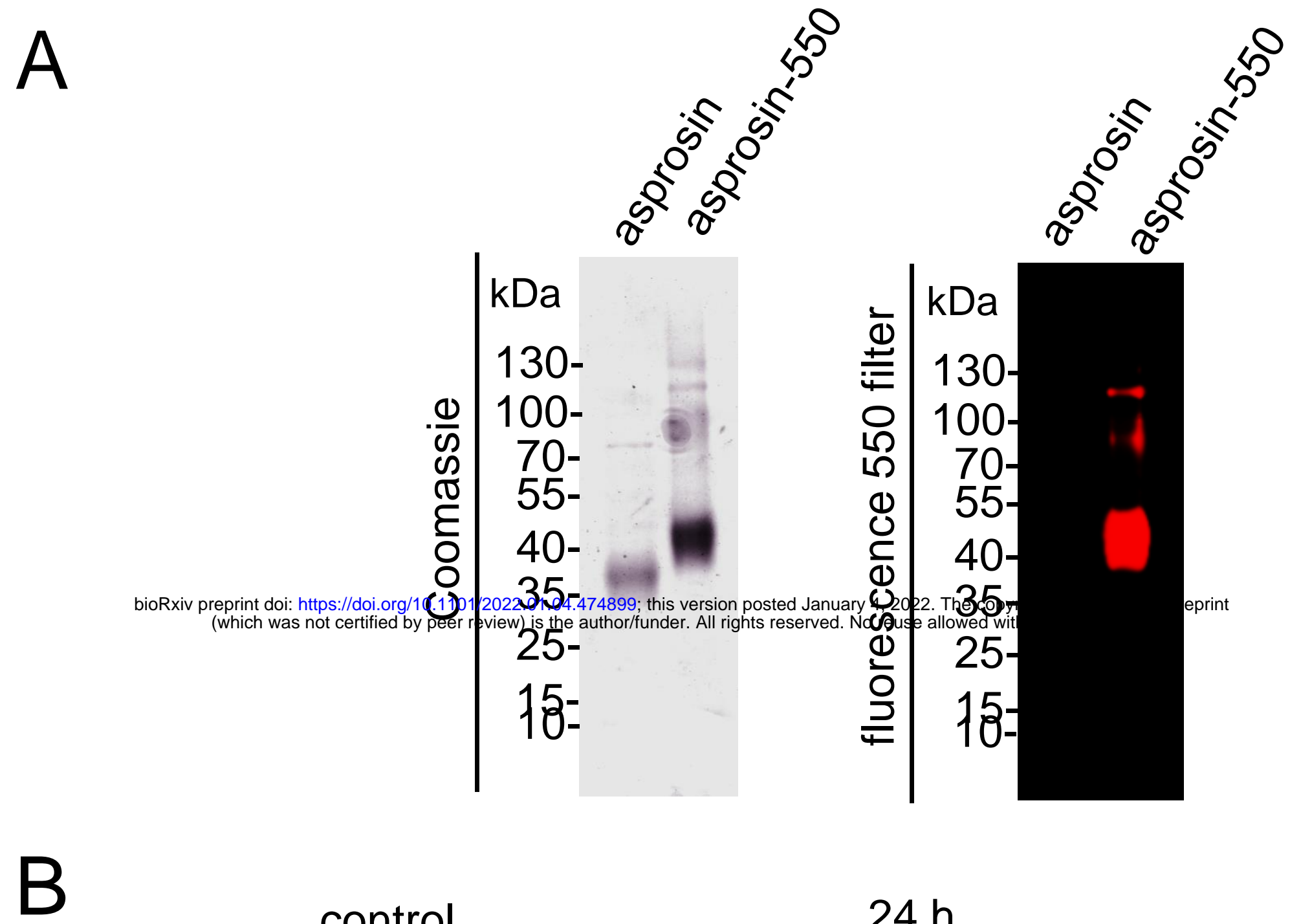

control

$24 \mathrm{~h}$

$48 \mathrm{~h}$
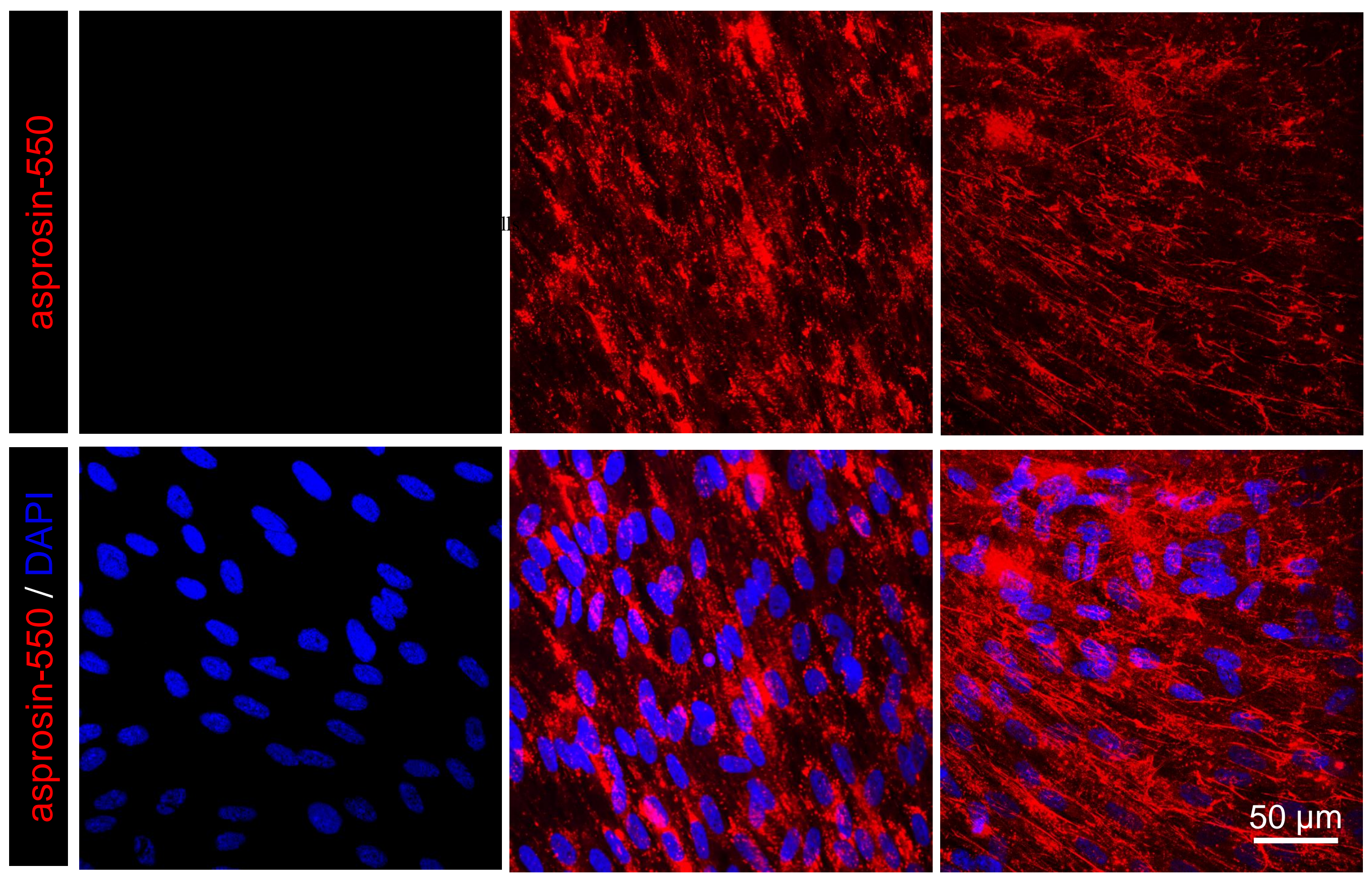

Supplementary Figure S1: Asprosin fiber formation upon administration of fluorescently labelled asprosin (Asprosin-550). A. (left) Coomassie stained quality control gel of Atto-550 labelled asprosin. (right) Visualization of Atto-550 fluorescent moiety which was conjugated to asprosin by ChemoStar Touch ECL \& Fluorescence Imager. B. Detection of fluorescent asprosin (red) and nuclei (DAPI in blue) after administration to RPE cells for $24 \mathrm{~h}$ and $48 \mathrm{~h}$ showing formation of asprosin positive fibers $48 \mathrm{~h}$ after asprosin-550 (5 $\mathrm{gg} / \mathrm{ml})$ administration. Images were obtained from a Leica SP8 confocal microscope and were processed using Leica LAS AF Lite 4.0 software and Fiji/lmageJ software to obtain average intensity Z-projection. 
hunan_asprosin hunan_placensin Consensus
1

20

30

STHETDASHIEDQSETEAHWSLASHDWEKTAIFAFHISHWSHKURILELLPHLTTLTHHHRTLIESGHED SIHEPDPTHWEISLESWDHDSPWHKFLSHLGSKEHILELRPAIQPLHHIRYYISQGHDD SnhEd\#peaea\# ! SLaSwDn \#kPannaFHiSHlgnKerILELrPHiqPLnHHnRYIIeqGH \#D

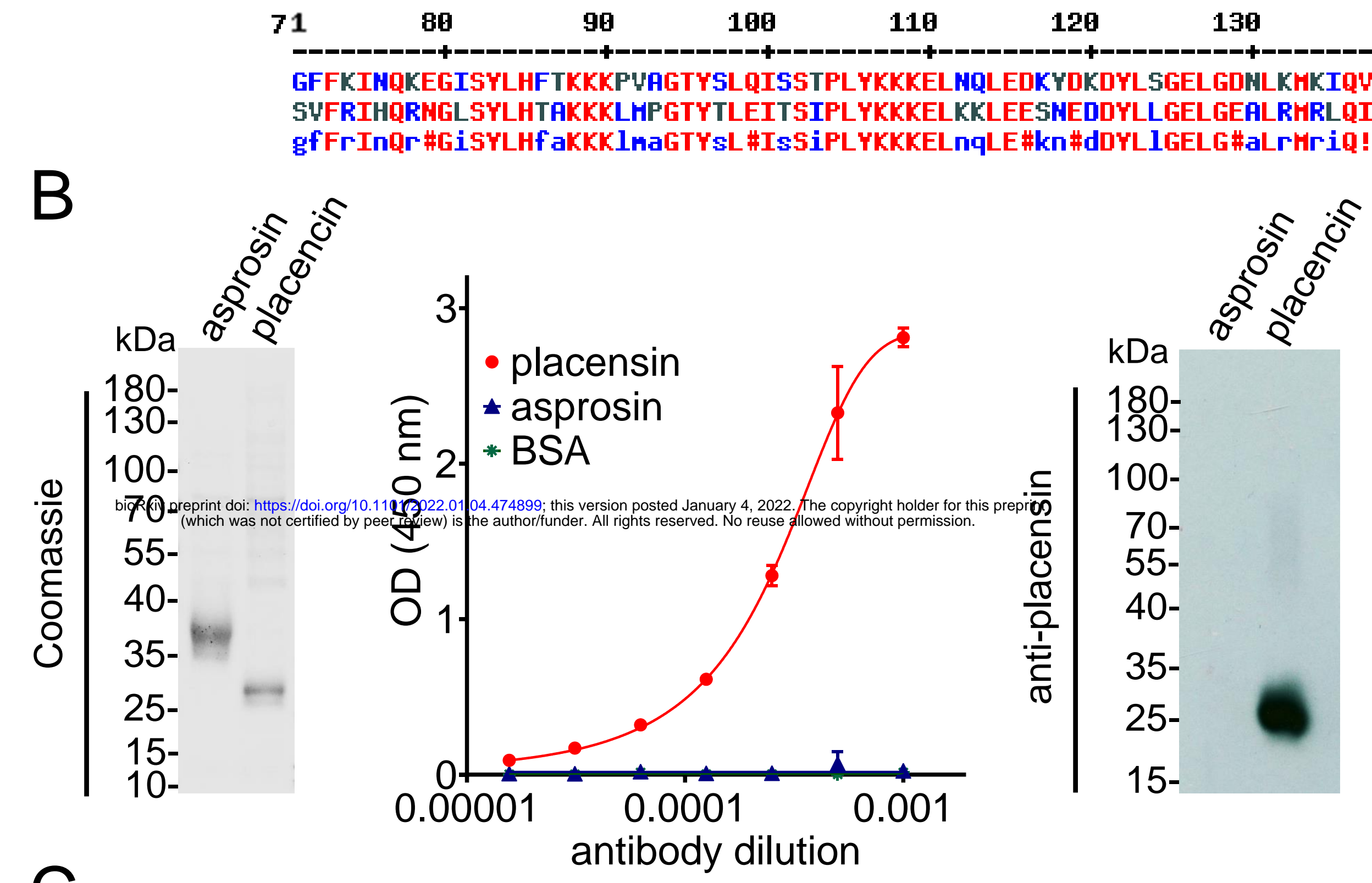

placensin
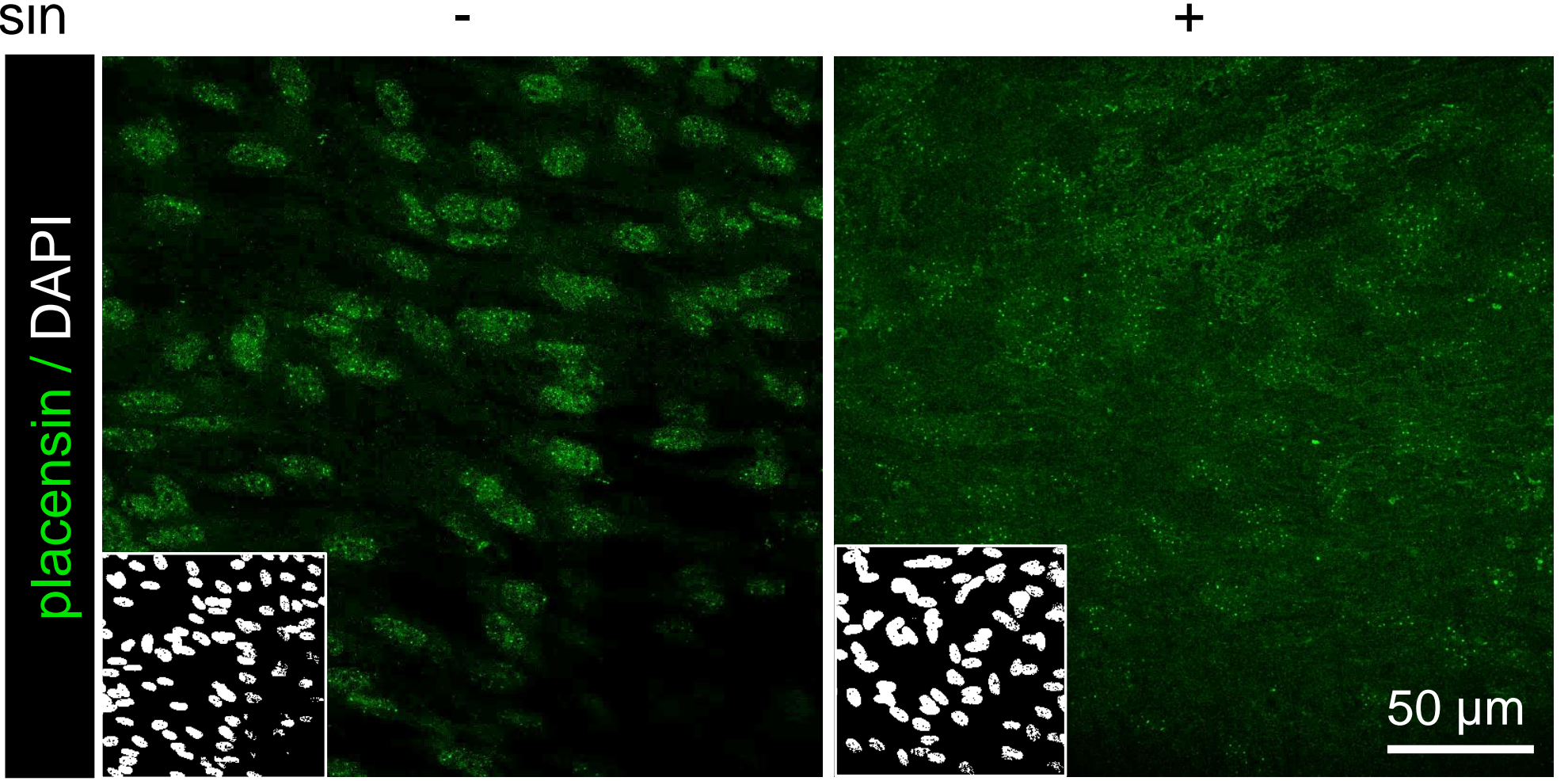

Supplementary Figure S2: Administration of placensin to RPE cells did not result in fiber formation. A. Sequence alignment of human asprosin and placensin using Multalin software showed $47.14 \%$ sequence homology between both proteins. B. (left) Coomassie stained quality control gel of recombinantly produced and purified (> $95 \%$ purity) asprosin and placensin after affinity chromatography. (middle) Affinity purified polyclonal human-placensin antibody raised in rabbit showed high specificity in in detecting coated placensin (100 ng/well) by direct ELISA. Data points represent mean \pm SD of duplicates. (right) Human placensin antibody specifically recognizes human placensin in western blot analysis. C. Recombinant placensin $(5 \mu \mathrm{g} / \mathrm{ml})$ was administered to RPE cells. $48 \mathrm{~h}$ after placensin treatment, cells were immunostained with human placensin antibody (green) and DAPI (white). Placensin immunostaining showed only intracellular signals. Images were obtained from a Leica SP8 confocal microscope and were processed using Leica LAS AF Lite 4.0 software and Fij//ImageJ software to obtain average intensity Z-projection. 
H._ASP. STHETDHSNIEDQSETEANYSLASHDYEKTAIFFFNISHYSNKYRILELLPALTTLTNHNRYLIESGNED

H_ASP. STHETDASDIQDGSEMEANYSLASHDYEKPASFAFNISHYNNKYRILELLPALTTLHNHNRYLIESGNED Consensus STHETDFS\#I\#DqSEnEANYSLASHDYEKpHiFAFNISHYnFKYRILELLPALTTLnHHNRYLIESGNED

\section{1}

80

90

100

110

120

130

140

GFFKIMQKEGISYLHFTKKKPUAGTYSLQISSTPLYKKKELNQLEDKYDKDYLSGELGDNLKMKIQYLLH GFFKINQKEGYSYLHFTKKKPWAGTYSLQISSTPLYKKKELNQLEDRYDKDYLSGELGDNLKHKIQILLH GFFKINQKEG ! SYLHFTKKKPWAGTYSLQISSTPLYKKKELNQLEDrYDKDYLSGELGDNLKMKIQ!LLH

$\mathrm{kDa}$

$180-$

$100-$

$70-$

$55-$

$40-$

35-

25-

$15-$

\section{murine asprosin}

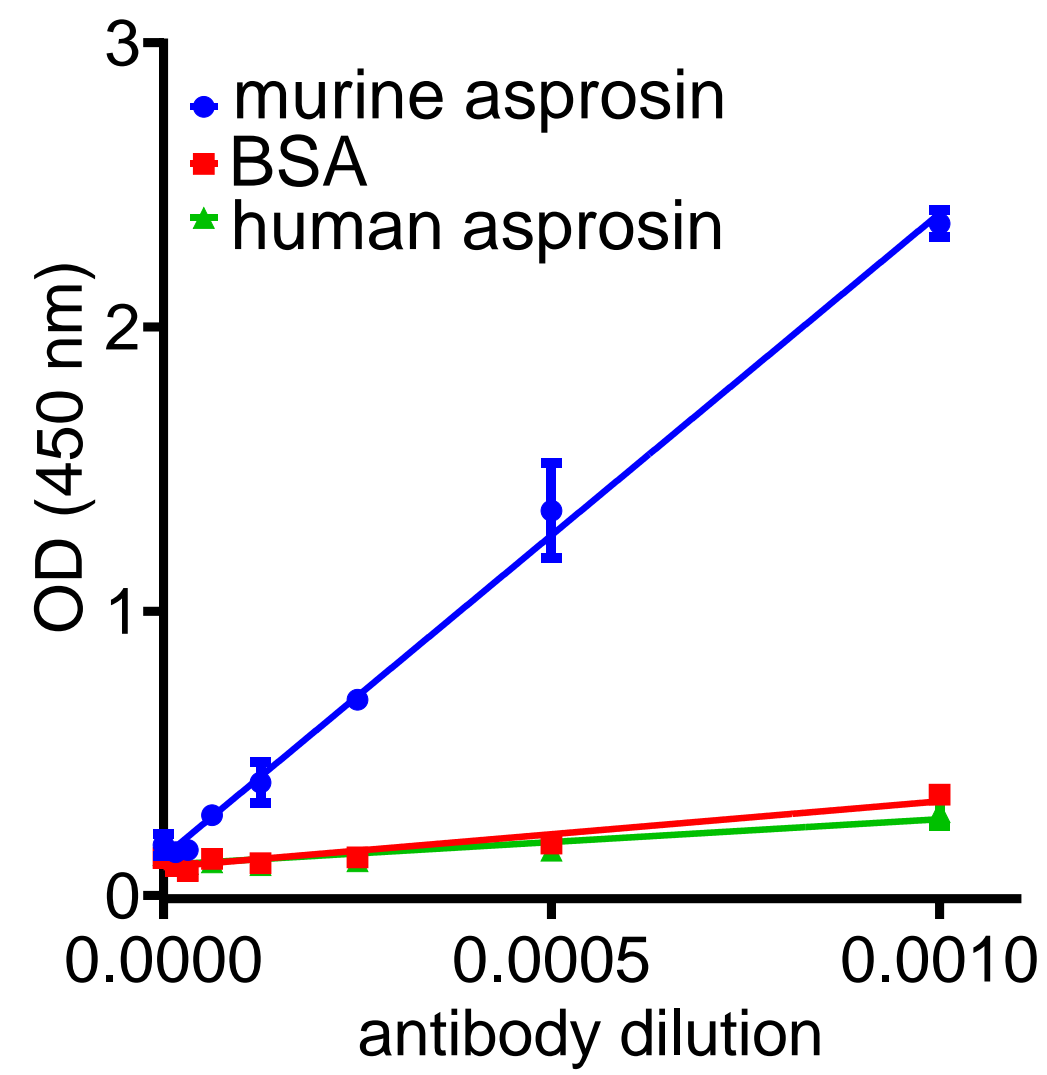

Supplementary Figure S3: Production polyclonal antibody specifically recognizing murine asprosin. A. Sequence alignment between human and murine asprosin using Multalin software showed $92.14 \%$ sequence homology between both proteins. B. Coomasie stained quality control gel of recombinantly produced and purified murine asprosin (> $95 \%$ purity) after affinity chromatography. C. Affinity purified polyclonal antibody raised in rabbit showed a high specificity in detecting coated murine asprosin (100 ng / well) by direct ELISA. Data points represent mean \pm SD of duplicates. 
